\title{
ANALYSIS OF LEAST SQUARES FINITE ELEMENT METHODS FOR THE STOKES EQUATIONS
}

\author{
PAVEL B. BOCHEV AND MAX D. GUNZBURGER
}

\begin{abstract}
In this paper we consider the application of least squares principles to the approximate solution of the Stokes equations cast into a first-order velocity-vorticity-pressure system. Among the most attractive features of the resulting methods are that the choice of approximating spaces is not subject to the LBB condition and a single continuous piecewise polynomial space can be used for the approximation of all unknowns, that the resulting discretized problems involve only symmetric, positive definite systems of algebraic equations, that no artificial boundary conditions for the vorticity need be devised, and that accurate approximations are obtained for all variables, including the vorticity. Here we study two classes of least squares methods for the velocity-vorticitypressure equations. The first one uses norms prescribed by the a priori estimates of Agmon, Douglis, and Nirenberg and can be analyzed in a completely standard manner. However, conforming discretizations of these methods require $C^{1}$ continuity of the finite element spaces, thus negating the advantages of the velocity-vorticity-pressure formulation. The second class uses weighted $L^{2}$-norms of the residuals to circumvent this flaw. For properly chosen meshdependent weights, it is shown that the approximations to the solutions of the Stokes equations are of optimal order. The results of some computational experiments are also provided; these illustrate, among other things, the necessity of introducing the weights.
\end{abstract}

\section{INTRODUCTION}

Recently, there has been substantial interest in the use of least squares principles for the approximate solution of the Navier-Stokes equations of incompressible flow; for some examples of bona fide least squares methods, one may consult, e.g., $[5,8,9,11,12,19,20,21,22,23,24,28]$. The computational results provided in these papers indicate that the methods considered are effective; however, careful analyses of these methods indicate that they do not yield optimally accurate approximations. According to the theory of [7], the formulation and analysis of discretization methods for the Stokes problem are critical for the understanding of like methods for the Navier-Stokes equations. Thus, the main goal of this paper is to analyze least squares methods for the Stokes problem.

Received by the editor June 8, 1993 and, in revised form, October 12, 1993.

1991 Mathematics Subject Classification. Primary 65N30, 65N12, 76M10.

This work was supported by the Air Force Office of Scientific Research under grant number AFOSR-93-1-0061. 
Here we develop two such methods that result in optimally accurate approximations. For the case of the important velocity boundary conditions, one method requires the introduction of mesh-dependent weights in the least squares functional in order to obtain optimal-order approximations using merely $C^{0}$ finite element spaces. The other method, which is not practical since it requires the use of $C^{1}$-finite element spaces, is introduced in order to show the proper formulation of a method for which the use of weights in the functional is not necessary. For the formulation of our methods we cast the Stokes equations into a first-order system involving the velocity, vorticity, and pressure as dependent variables. In two dimensions, one has four unknown scalar fields, and in three dimensions their number increases to seven. However, the order of differentiation in each variable is one, so that there exists the possibility of discretizing the least squares minimization problem using merely continuous finite element spaces. The validity of this argument is one of the central subjects of this paper.

Although there are other ways to cast the Stokes problem into a first-order system (see, e.g., [3] and [5]), for several reasons we prefer to work with the velocity-vorticity-pressure equations. First, we can directly approximate the vorticity variable. Also, the velocity-vorticity-pressure equations involve fewer variables. Lastly, there is a large group of standard finite element methods which use the vorticity as a primary variable and can be used for comparison with our methods.

Least squares methods for elliptic boundary value problems of order $2 m$ were studied in [6]. More recently, a least squares theory for elliptic systems of Agmon, Douglis, and Nirenberg type was developed in [2] and, in particular, the primitive variable Stokes problem was treated within this theory. Least squares methods for Petrovskii (see [27]) elliptic systems in the plane were considered in [29]. Least squares ideas have also been used for stabilization of standard saddle-point formulations of flow and elasticity problems; for results in this direction the reader may consult [3] and [15].

Compared to the classical mixed Galerkin formulation (see, e.g., [16] or [17]), the least squares methods considered here offer certain advantages, especially for large-scale computations. For example,

the choice of approximating spaces is not subject to the $L B B$ condition, and a single continuous piecewise polynomial space can be used for the approximation of all unknowns

and

its application to the Navier-Stokes equations together with, for example, a Newton linearization, results in symmetric, positive definite, linear algebraic systems, at least in a neighborhood of the solution.

Thus, used in conjunction with a properly implemented continuation (with respect to the Reynolds number) technique, the method will only encounter symmetric, positive definite, linear systems in the solution procedure. The solution of these systems can be accomplished effectively by, e.g., conjugate gradient methods. As a result,

a method can be devised which requires no matrix assembly, even at the element level. 
This is particularly important for large-scale computations since standard Galerkin mixed methods produce nonsymmetric systems, which must then be solved by direct methods or by complex and nonrobust iterative methods. We also mention two other advantages of the least squares approach considered here. The first is that

no artificial boundary conditions for the vorticity need be devised at boundaries where the velocity is specified.

The second is that, unlike many other methods involving the vorticity, e.g., see [14] and [18],

\section{accurate vorticity and pressure approximations are obtained.}

The determination of the proper function spaces in which boundary value problems for the velocity-vorticity-pressure equations are well posed is crucial to the success of the methods considered here. The crucial issue is the choice of spaces for the dependent variables and the data that make the (elliptic) differential operator compatible with the boundary operator. If, for example, we assume an equal order of differentiability for all unknowns (as it may seemingly look appropriate for a first-order system), then compatibility of a first-order elliptic operator in the plane with a given boundary operator can be established by verifying the Lopatinskii condition [29]. However, if the velocity-vorticitypressure equations in two dimensions are supplemented with velocity boundary conditions, then the Lopatinskii condition does not hold. This is not surprising if one recalls that the vorticity is defined as the curl of the velocity and thus should not, in general, have the same order of differentiability. For some boundary conditions, e.g., prescribing the normal component of the velocity and the pressure, the Lopatinskii condition is satisfied, and such cases can be treated with the least squares theory developed in [29].

In order to formulate and study least squares methods with the important velocity boundary conditions and in three space dimensions, we need the more general theory of Agmon-Douglis-Nirenberg (ADN) [1]. This theory permits, even for a first-order system, to assume different orders of differentiability for the unknowns by assigning indices to each equation and unknown function. An elliptic boundary value problem is then considered to be well posed if it is possible to find a set of indices under which the Complementing Condition of [1] holds. These indices, if they exist, determine the proper function spaces for both the data and the solution of the boundary value problem. Whenever all unknowns are assigned the same index, the Complementing Condition for firstorder systems in the plane is equivalent to the Lopatinskii condition; see [29]. In the three-dimensional case, although there is no equivalent to the Lopatinskii condition, the velocity boundary condition poses the same problem: if an equal order of differentiability is assumed for all seven unknowns, then the Complementing Condition does not hold.

Given a well-posed elliptic boundary value problem, we define the standard least squares functional to be the sum of the residuals of the equations measured in norms determined by the ADN index of the corresponding equation. The minimization of this functional is equivalent to solving a variational problem. Coercivity of this problem follows from the ADN a priori estimates, and its error analysis can be carried out in a completely standard manner. However, for each 
nonzero equation index, the standard functional will include norms stronger than the $L^{2}$-norm. Hence, a conforming discretization of such a least squares principle will require the use of continuously differentiable finite element spaces; this is a serious practical flaw.

To circumvent this flaw, we introduce a mesh-dependent least squares functional where the residual of each equation is measured in the $L^{2}$-norm multiplied by a weight determined by the equation index and the mesh parameter $h$. The purpose of these weights is to modify the behavior of the $L^{2}$-norm terms so that they now resemble, as $h$ tends to zero, the behavior of the stronger norms prescribed originally by the ADN equation indices. A single piecewise polynomial finite element space which is merely continuous can now be used for all test and trial functions at the price of a more elaborate error analysis than in the standard case. The mesh-dependent least squares functional considered here is similar to the one in [2]. However, the latter is based on the primitive variable formulation of the Stokes problem and thus necessarily requires $C^{1}$ spaces for the conforming approximation of the velocity field.

The paper is organized as follows. For brevity we state and prove most of the results for the two-dimensional case. In $\S 2$, we introduce the velocity-vorticitypressure equations and analyze the Complementing Condition for two different choices of boundary operators. Then, we extend the ADN a priori estimates for the first-order system to negative regularity indices. In $\S 3$, we introduce the standard least squares principle and show that optimal convergence rates can be achieved for conforming discretizations. The mesh-dependent least squares functional is introduced in $\S 4$. We show that for properly chosen weights, the minimization of this functional produces approximations which converge to smooth solutions of the Stokes equations at the best possible rate. In $\S 5$, we consider least squares methods for three-dimensional problems. Most of the results for the three-dimensional setting can be carried over from the corresponding two-dimensional results and, thus, we focus on the differences between the formulation and analysis of such methods in three dimensions and their twodimensional counterparts. In $\S 6$ we present some numerical results obtained for the two different boundary operators. The first operator corresponds to the velocity boundary condition and requires weights in the least squares functional. The importance of these weights is assessed by comparing the numerical results obtained with and without the weights. The second operator which satisfies the Lopatinskii condition and corresponds to the normal velocity-pressure boundary condition, provides an example of conforming discretizations of the standard least squares principle that result in a practical method.

\section{The Velocity-Vorticity-PRESSURE StOKES EQUATIONS}

Let $\Omega \in \mathbf{R}^{2}$ be an open and bounded set with smooth boundary $\Gamma$. The Stokes equations are given by

$$
\begin{aligned}
-\Delta \mathbf{u}+\operatorname{grad} p=\mathbf{f}_{1} & \text { in } \Omega, \\
\operatorname{div} \mathbf{u}=0 & \text { in } \Omega,
\end{aligned}
$$

where $\mathbf{u}=\left(u_{1}, u_{2}\right)$ denotes the velocity, $p$ the pressure, and $\mathbf{f}_{1}$ the body force. The system (1) is a uniformly elliptic system of total order 4 . We recall that in 
two dimensions we have two curl operators:

$$
\operatorname{curl} \omega=\left(\begin{array}{c}
\omega_{y} \\
-\omega_{x}
\end{array}\right), \quad \operatorname{curl} \mathbf{u}=u_{2 x}-u_{1 y} .
$$

The vorticity $\omega$ is defined by $\omega=$ curl $\mathbf{u}$. Using the identity curl curl $\mathbf{u}=$ $-\Delta \mathbf{u}+\operatorname{grad} \operatorname{div} \mathbf{u}$, and in view of the incompressibility constraint $\operatorname{div} \mathbf{u}=0$, one may replace the first equation in $(1)$ by curl $\omega+\operatorname{grad} p=\mathbf{f}_{1}$. Let $U=(\omega, p, \mathbf{u})$. Then, the generalized velocity-vorticity-pressure form of the Stokes problem is

$$
\begin{gathered}
\mathscr{L} U=\left(\begin{array}{ccc}
\text { curl } & \operatorname{grad} & 0 \\
-1 & 0 & \operatorname{curl} \\
0 & 0 & \operatorname{div}
\end{array}\right)\left(\begin{array}{l}
\omega \\
p \\
\mathbf{u}
\end{array}\right)=\left(\begin{array}{l}
\mathbf{f}_{1} \\
f_{2} \\
f_{3}
\end{array}\right)=F \quad \text { in } \Omega, \\
\mathscr{R} U=G \quad \text { on } \Gamma .
\end{gathered}
$$

Here we examine two choices for the boundary operators. The first one imposes the velocity on the boundary, i.e.,

$$
\mathscr{R}_{1} U=\left(\begin{array}{l}
u_{1}^{0} \\
u_{2}^{0}
\end{array}\right) \quad \text { on } \Gamma,
$$

where $\mathbf{u}^{0}$ is a given function defined along $\Gamma$. The second boundary operator imposes the pressure and the normal component of velocity, i.e.,

$$
\mathscr{R}_{2} U=\left(\begin{array}{c}
P^{0} \\
U^{0}
\end{array}\right) \quad \text { on } \Gamma,
$$

where $P^{0}$ and $U^{0}$ are given functions defined along $\Gamma$. For the solvability of the boundary value problems (2)-(4) and (2)-(5), the data must be subject to the compatibility conditions

$$
\int_{\Omega} f_{3} d x=\int_{\Gamma} \mathbf{u}^{0} \cdot \mathbf{n} d s
$$

and $\int_{\Omega} f_{3} d x=\int_{\Gamma} U^{0} d s$, respectively.

The boundary conditions (5) are considered here mostly because they satisfy the Lopatinskii condition, and accordingly the standard least squares approach will result in a practical method which can be analyzed in a familiar way. The boundary conditions (4) are more difficult to analyze and have presented serious problems in the development of effective computational methods involving the vorticity as a dependent variable; see, e.g., [18]. Here we shall only consider homogeneous boundary conditions that are satisfied exactly by candidate solutions and their finite-dimensional approximations. Although less general, compared with the inclusion of inhomogeneous boundary conditions into the least squares functional, this setting eliminates some nonessential details from the error analysis. At the price of some tedious calculations, our results can be extended to the more general case. Indeed, another potential advantage of the least squares approach is that boundary conditions could be enforced in a weak sense through their inclusion in the least squares functional (see [2, 6, 29]).

If $f_{2}=f_{3}=0$, the boundary value problem (2) and (4) is equivalent to the Stokes problem (1) and (4) in primitive variable form. In order to guarantee the uniqueness of solutions of (2) and (4), one also has to impose an additional 
constraint on the pressure. The usual choice is to require that the pressure have zero mean over $\Omega$, i.e.,

$$
\int_{\Omega} p d x=0
$$

With this assumption we can prove the following result.

Proposition 1. The problem (2), (4), and (7) has a unique solution for all smooth data $\mathbf{f}_{1}, f_{2}, f_{3}$, and $\mathbf{u}_{0}$.

Proof. Let $\omega, p$, and $\mathbf{u}$ be smooth functions which satisfy

$$
\begin{aligned}
& \operatorname{curl} \omega+\operatorname{grad} p=0 \quad \text { in } \Omega, \\
& \operatorname{curl} \mathbf{u}-\omega=0 \text { in } \Omega \text {, } \\
& \operatorname{div} \mathbf{u}=0 \quad \text { in } \Omega, \\
& \mathbf{u}=0 \text { on } \Gamma \text {. }
\end{aligned}
$$

The first equation in (8) implies that $\omega$ and $p$ must be harmonic functions. Taking the Laplacian of the second equation yields

$$
\Delta \operatorname{curl} \mathbf{u}=\operatorname{curl}(\Delta \mathbf{u})=\Delta \omega=0 .
$$

Let us suppose that $\Delta \mathbf{u}=0$; then, the boundary condition $\mathbf{u}=0$ on $\Gamma$ implies that $\mathbf{u} \equiv 0$. If, on the other hand, we suppose that $\Delta \mathbf{u} \neq 0$, the identity $\operatorname{curl}(\Delta \mathbf{u})=0$ implies that $\Delta \mathbf{u}$ must be a gradient, i.e., $\Delta \mathbf{u}=\operatorname{grad} q$ for some $q$. Then the pair $(\mathbf{u}, q)$ solves the homogeneous Stokes problem

$$
\begin{aligned}
& -\Delta \mathbf{u}+\operatorname{grad} q=0 \quad \text { in } \Omega, \\
& \operatorname{div} \mathbf{u}=0 \quad \text { in } \Omega, \\
& \mathbf{u}=0 \text { on } \Gamma \text {, }
\end{aligned}
$$

and we can infer that $\mathbf{u} \equiv 0$. Now, from the second equation in (8), it follows that $\omega=\operatorname{curl} \mathbf{u} \equiv 0$ and then from the first equation we have that $\operatorname{grad} p=0$. Then, (7) implies that $p=0$.

Uniqueness for the problem (2)-(5), (7) can be established in a similar way.

Let us now define the necessary function spaces. We use $\mathscr{D}(\Omega)$ to denote the space of smooth functions with compact support in $\Omega$ and $\mathscr{D}(\bar{\Omega})$ to denote the restrictions of the functions in $\mathscr{D}\left(\mathbf{R}^{n}\right)$ on $\bar{\Omega}$. For $s \geq 0$ we use the standard notation and definition for the Sobolev spaces $H^{s}(\Omega)$ and $H^{s}(\Gamma)$ with inner products and norms denoted by $(\cdot, \cdot)_{s, \Omega}$ and $(\cdot, \cdot)_{s, \Gamma}$ and $\|\cdot\|_{s, \Omega}$ and $\|\cdot\|_{s, \Gamma}$, respectively. Often, when there is no chance for confusion, we will omit the domain $\Omega$ from the inner product and norm designation.

As usual, $H_{0}^{s}(\Omega)$ will denote the closure of $\mathscr{D}(\Omega)$ with respect to the norm $\|\cdot\|_{s, \Omega}$, and $L_{0}^{2}(\Omega)$ will denote the subspace of square integrable functions with zero mean. We set $\widetilde{\mathscr{D}}(\Omega)=\mathscr{D}(\Omega) \cap L_{0}^{2}(\Omega), \widetilde{\mathscr{D}}(\bar{\Omega})=\mathscr{D}(\bar{\Omega}) \cap L_{0}^{2}(\Omega)$, and $\widetilde{H}^{s}(\Omega)=H^{s}(\Omega) \cap L_{0}^{2}(\Omega)$. For negative values of $s$ the spaces $H^{s}(\Omega)$, $H_{0}^{s}(\Omega)$, and $\widetilde{H}^{s}(\Omega)$ are defined as the closures of $\mathscr{D}(\bar{\Omega}), \mathscr{D}(\Omega)$, and $\widetilde{\mathscr{D}}(\bar{\Omega})$ with respect to the norm

$$
\|\phi\|_{s}=\sup _{q \in D(\Omega)} \frac{\int_{\Omega} \phi q d x}{\|q\|_{-s, \Omega}},
$$


where $D(\Omega)=\mathscr{D}(\bar{\Omega}), \mathscr{D}(\Omega)$, and $\widetilde{D}(\bar{\Omega})$, respectively. We identify $H^{s}(\Omega)$, $H_{0}^{s}(\Omega)$, and $\widetilde{H}^{s}(\Omega)$ with the duals of $H^{-s}(\Omega), H_{0}^{-s}(\Omega)$, and $\widetilde{H}^{-s}(\Omega)$, respectively; for $s \in \mathbf{R}$ these spaces form interpolating families. By $(\cdot, \cdot)_{\left(s_{1}, \ldots, s_{n}\right)}$ and $\|\cdot\|_{\left(s_{1}, \ldots, s_{n}\right)}$ we denote inner products and norms, respectively, on the product spaces $H^{s_{1}}(\Omega) \times \cdots \times H^{s_{n}}(\Omega)$; when all $s_{i}$ are equal, we shall simply write $(\cdot, \cdot)_{s, \Omega}$ and $\|\cdot\|_{s, \Omega}$.

2.1. The Agmon-Douglis-Nirenberg estimates. Let $\mathscr{L}=\left\{\mathscr{L}_{i j}\right\}, i, j=$ $1, \ldots, N$, denote an elliptic differential operator of order $2 m$ and $\mathscr{R}=\left\{\mathscr{R}_{l j}\right\}$, $l=1, \ldots, m, j=1, \ldots, N$, denote a boundary operator. We consider the boundary value problem

$$
\begin{array}{ll}
\mathscr{L}(\mathbf{x}) U=F & \text { in } \Omega, \\
\mathscr{R}(\mathbf{x}) U=G & \text { on } \Gamma .
\end{array}
$$

Following [1], we assign a system of integer indices $\left\{s_{i}\right\}, s_{i} \leq 0$, for the equations and $\left\{t_{j}\right\}, t_{j} \geq 0$, for the unknown functions such that the order of $\mathscr{L}_{i j}$ is bounded by $s_{i}+t_{j}$. Then, the principal part $\mathscr{L}^{p}$ of $\mathscr{L}$ is defined as all those terms $\mathscr{L}_{i j}$ with orders exactly equal to $s_{i}+t_{j}$. The principal part $\mathscr{R}^{p}$ is defined in a similar way by assigning nonpositive weights $r_{l}$ to each row in $\mathscr{R}$ such that the order of $\mathscr{R}$ is bounded by $r_{l}+t_{j}$.

The Complementing Condition [1] is a local algebraic condition on the principal parts $\mathscr{L}^{p}$ and $\mathscr{R}^{p}$ of the differential and boundary operators which guarantees the compatibility of a particular set of boundary conditions with the given system of differential equations. This condition is necessary and sufficient for coercivity estimates to be valid; see [1]. Before introducing the Complementing Condition, some notation must be established.

Let $P$ be any point on the boundary $\Gamma$ and let $\mathbf{n}$ be the unit outer normal vector to $\Gamma$ at $P$. Let $\xi$ be any nonzero vector tangent to $\Gamma$ at $P$. Let $\mathscr{L}^{\prime}$ denote the adjoint matrix to $\mathscr{L}^{p}$. We first require that the following condition is satisfied.

Supplementary Condition on $\mathscr{L}$. First, $\operatorname{det} \mathscr{L}^{p}(\xi)$ is of even degree $2 m$ (with respect to $\boldsymbol{\xi})$. Also, for every pair of linearly independent real vectors $\boldsymbol{\xi}, \boldsymbol{\xi}^{\prime}$, the polynomial $\operatorname{det} \mathscr{L}^{p}\left(\boldsymbol{\xi}+\tau \boldsymbol{\xi}^{\prime}\right)$ in the complex variable $\tau$ has exactly $m$ roots with positive imaginary part.

For any elliptic system in three or more dimensions, the Supplementary Condition is satisfied, i.e., the characteristic equation $\operatorname{det} \mathscr{L}^{p}(\boldsymbol{\xi}+\tau \mathbf{n})=0$ always has exactly $m$ roots with positive imaginary parts. In two dimensions, this condition must be verified for any given $\mathscr{L}^{p}$.

Let $\tau_{k}^{+}(\boldsymbol{\xi})$ denote the $m$ roots of $\operatorname{det} \mathscr{L}^{p}\left(\boldsymbol{\xi}+\tau \boldsymbol{\xi}^{\prime}\right)$ having positive imaginary part. Let

$$
M^{+}(\boldsymbol{\xi}, \tau)=\prod_{k=1}^{m}\left(\tau-\tau_{k}^{+}(\boldsymbol{\xi})\right) .
$$

Then, we have the following definition [1].

Complementing Condition. For any point $P \in \Gamma$ and any real, nonzero vector $\xi$ tangent to $\Gamma$ at $P$, regard $M^{+}(\xi, \tau)$ and the elements of the matrix

$$
\sum_{j=1}^{N} \mathscr{R}_{l j}^{p}(\boldsymbol{\xi}+\tau \mathbf{n}) \mathscr{L}_{j k}^{\prime}(\boldsymbol{\xi}+\tau \mathbf{n})
$$


as polynomials in $\tau$. The operators $\mathscr{L}$ and $\mathscr{R}$ satisfy the Complementing Condition if the rows of the latter matrix are linearly independent modulo $M^{+}(\xi, \tau)$, i.e.,

$$
\sum_{l=1}^{m} C_{l} \sum_{j=1}^{N} \mathscr{R}_{l j}^{p} \mathscr{L}_{j k}^{\prime} \equiv 0 \quad\left(\bmod M^{+}\right)
$$

if and only if the constants $C_{l}$ all vanish.

In [1], the following result is proved.

Theorem 1. Assume that the system (10) is uniformly elliptic (and in $2 D$ satisfies the Supplementary Condition) and assume that the boundary conditions (11) satisfy the Complementing Condition. Furthermore, assume that for some $q \geq 0$, $U \in \prod_{j=1}^{N} H^{q+t_{j}}(\Omega), F \in \prod_{i=1}^{N} H^{q-s_{i}}(\Omega)$, and $G \in \prod_{l=1}^{m} H^{q-r_{l}-1 / 2}(\Gamma)$. Then, there exists a constant $C>0$ such that

$$
\sum_{j=1}^{N}\left\|u_{j}\right\|_{q+t_{j}, \Omega} \leq C\left(\sum_{i=1}^{N}\left\|F_{i}\right\|_{q-s_{i}, \Omega}+\sum_{l=1}^{m}\left\|G_{l}\right\|_{q-r_{l}-1 / 2, \Gamma}+\sum_{j=1}^{N}\left\|u_{j}\right\|_{0, \Omega}\right)
$$

Moreover, if the problem (10)-(11) has a unique solution, then the $L^{2}$ norm on the right-hand side of (13) can be omitted.

The Complementing Condition rules out existence of wildly oscillating solutions which decay exponentially away from the boundary. Indeed, let us suppose that in a neighborhood of $P$ the boundary $\Gamma$ is flattened so that it lies on the plane $z=0$. Then, on $z \geq 0$ we consider a homogeneous, constantcoefficient (frozen at $P$ ) system of partial differential equations corresponding to the principal part of the original system (10) with homogeneous (also constant-coefficient) boundary conditions corresponding to the principal part of the boundary operator (11):

$$
\begin{array}{ll}
\mathscr{L}^{p}(P) U=0 & \text { in } z>0, \\
\mathscr{R}^{p}(P) U=0 & \text { on } z=0 .
\end{array}
$$

Now, let $\mathbf{x}=(x, y, 0)$ and $\xi$ be any real vector in the plane $z=0$. The Complementing Condition requires that all solutions to (14)-(15) of the form $\mathbf{u}=e^{i \mathbf{x} \cdot \xi} \mathbf{v}(z)$ must be identically zero, i.e., $\mathbf{v} \equiv 0$. The ansatz $\mathbf{u}=e^{i \mathbf{x} \cdot \boldsymbol{\xi}} \mathbf{v}(z)$ reduces (14)-(15) to a system of ordinary differential equations for $\mathbf{v}$, which provides for an alternative way (see [26]) to verify the Complementing Condition.

2.1.1. The Complementing Condition for the velocity-vorticity-pressure equations. In this subsection we discuss the Complementing Condition for the twodimensional Stokes equations in velocity-vorticity-pressure form (2) with the velocity boundary conditions (4).

Let us first assume equal order of differentiability for all unknown functions. Then we have to choose the indices for the equations and unknowns according to $s_{1}=s_{2}=s_{3}=s_{4}=0$ and $t_{1}=t_{2}=t_{3}=t_{4}=1$. The symbol of the principal 
part of (2), according to these indices, is

$$
\mathscr{L}^{p}(\boldsymbol{\xi})=\left(\begin{array}{cccc}
\xi_{2} & \xi_{1} & 0 & 0 \\
-\xi_{1} & \xi_{2} & 0 & 0 \\
0 & 0 & -\xi_{2} & \xi_{1} \\
0 & 0 & \xi_{1} & \xi_{2}
\end{array}\right) .
$$

The determinant of the principal part is $\operatorname{det} \mathscr{L}^{p}(\boldsymbol{\xi})=\operatorname{det} \mathscr{L}(\xi)=-\left(\xi_{1}^{2}+\xi_{2}^{2}\right)^{2}=$ $-|\xi|^{4}$, and hence the uniform ellipticity condition

$$
C_{e}^{-1}|\xi|^{2 m} \leq\left|\operatorname{det} \mathscr{L}^{p}(\xi)\right| \leq C_{e}|\xi|^{2 m}
$$

holds with $m=2$ and $C_{e}=1$. It is easy to see that $\mathscr{L}^{p}$ also satisfies the Supplementary Condition.

Let $\boldsymbol{\xi}$ be a tangent vector to $\Gamma$; for simplicity, let $|\boldsymbol{\xi}|=1$ and $|\mathbf{n}|=1$. Without loss of generality we may assume that the coordinate axes are aligned with the directions of $\boldsymbol{\xi}$ and $\mathbf{n}$, so that $\boldsymbol{\xi}=(1,0)$ and $\mathbf{n}=(0,-1)$. Then, (12) reduces to

$$
-C_{1} \tau+C_{2}=(\tau-i) A_{1}, \quad C_{1}-C_{2} \tau=(\tau-i) A_{2},
$$

where $A_{1}$ and $A_{2}$ are arbitrary constants. Evidently, for $C_{1}=i, C_{2}=1$ and $A_{1}=i, A_{2}=-1$ the above identities hold, and therefore the Complementing Condition is not satisfied.

Remark 1. This conclusion is not surprising, for if we assume an equal order of differentiability for all unknowns, then the term $(-\omega)$ is not in the principal part of $\mathscr{L}$ and the system corresponding to (14)-(15) decouples into the two independent systems:

$$
\operatorname{curl} \omega+\operatorname{grad} p=0
$$

and

$$
\operatorname{curl} \mathbf{u}=0, \quad \operatorname{div} \mathbf{u}=0
$$

with boundary conditions solely on $\mathbf{u}$. Now, it is easy to see that for any $n$ the functions

$$
\omega_{n}=-\sin (n x) \exp (-n y), \quad p_{n}=\cos (n x) \exp (-n y)
$$

satisfy the first equation, decay exponentially away from the boundary, but $\left|\omega_{n}\right|_{1, \Omega} /\left\|\omega_{n}\right\|_{0, \Omega}=O(n)$.

Let us now show that if we assume different orders of differentiability for the unknown functions, then the Complementing Condition will hold for the velocity boundary condition. We now choose the following indices: $s_{1}=s_{2}=0$, $s_{3}=s_{4}=-1$ and $t_{1}=t_{2}=1, t_{3}=t_{4}=2$. The symbol of the corresponding principal part of (2) is then given by

$$
\mathscr{L}^{p}(\boldsymbol{\xi})=\left(\begin{array}{cccc}
\xi_{2} & \xi_{1} & 0 & 0 \\
-\xi_{1} & \xi_{2} & 0 & 0 \\
-1 & 0 & -\xi_{2} & \xi_{1} \\
0 & 0 & \xi_{1} & \xi_{2}
\end{array}\right) .
$$

As before, we find that $\operatorname{det} \mathscr{L}^{p}=-\left(\xi_{1}^{2}+\xi_{2}^{2}\right)^{2}=-|\xi|^{4}$, and thus the uniform ellipticity condition and the Supplementary Condition clearly hold. Again, without loss of generality, we may assume that the coordinate axes are chosen so 
that $\boldsymbol{\xi}=(1,0)$ and $\mathbf{n}=(0,-1)$. Then, (12) can be reduced to

$$
\begin{aligned}
-C_{1} \tau^{2}-C_{2} \tau & =A_{1}(\tau-i)^{2}, \\
-C_{1} \tau-C_{2} & =A_{2}(\tau-i)^{2} .
\end{aligned}
$$

The right-hand side of (19) is a second-degree polynomial, and equality is possible if and only if $A_{2}=C_{1}=C_{2} \equiv 0$. Hence, the Complementing Condition holds.

For smooth solutions $\omega, p$, and $\mathbf{u}$ of the boundary value problem (2)(4) and (7) this fact and the uniqueness result from Proposition 1 imply the following form of the a priori estimate (13):

$$
\|\omega\|_{q+1}+\|p\|_{q+1}+\|\mathbf{u}\|_{q+2} \leq C\left(\left\|\mathbf{f}_{1}\right\|_{q}+\left\|f_{2}\right\|_{q+1}+\left\|f_{3}\right\|_{q+1}\right),
$$

where $q \geq 0$. When $\omega \in H^{q+1}(\Omega), p \in \tilde{H}^{q+1}(\Omega)$, and $\mathbf{u} \in H^{q+2}(\Omega)^{2}$ are solutions of (2)-(4) for $\mathbf{f}_{1} \in H^{q}(\Omega)^{2}, f_{2} \in H^{q+1}(\Omega), f_{3} \in \widetilde{H}^{q+1}(\Omega), u_{1}^{0}=$ $u_{2}^{0}=0$, the estimate (20) follows by a density argument.

Remark 2 . One can verify that the boundary operator (5) satisfies the Complementing Condition with either choice (16) or (17) for the principal part.

Remark 3. The equal order of differentiability is implicitly assumed in the Lopatinskii condition. Indeed, this condition requires one to cast the first-order system into the canonical form $U_{x}+B U_{y}+C U+F=0$ and then to verify an algebraic condition involving only the matrix $B$ and the symbol of $\mathscr{R}$. Consequently, the boundary value problem (2)-(4) cannot be treated within the least squares theory of [29].

The analysis of least squares methods with mesh-dependent functionals requires that the estimate (20) be extended to negative regularity indices $q$. Results of this type hinge on the existence of a complete set of isomorphisms for the particular elliptic system. For example, a complete set of isomorphisms for Petrovskii systems is established in [27]. However, the problem (2)-(4), (7) is not of Petrovskii type. Nevertheless, we can still extend (20) for $q<0$ using the idea of [27] of passing to the adjoint equation, using the observation that after a suitable permutation of the equations the problem (2)-(4), (7) becomes selfadjoint.

Theorem 2. Let $U=(\omega, p, \mathbf{u}) \in D=\mathscr{D}(\overline{\mathbf{\Omega}}) \times \widetilde{\mathscr{D}}(\overline{\mathbf{\Omega}}) \times \mathscr{D}(\overline{\mathbf{\Omega}})^{2}, \mathbf{u}=0$ on $\Gamma$, and let $\mathbf{f}_{1}, f_{2}$, and $f_{3}$ be defined by (2). Then, the a priori estimate (20) holds for all $q \in \mathbf{R}$.

Proof. For the proof of this theorem we shall assume that $\mathscr{L}$ corresponds to the system (2) where div is replaced by - div and the equations are permuted so that the first one becomes the last one. We introduce the product spaces

$$
\begin{aligned}
X_{s} & =H^{s+1}(\Omega) \times \widetilde{H}^{s+1}(\Omega) \times\left[H^{s+2}(\Omega)\right]^{2}, & & s \geq 0, \\
Y_{s} & =H^{s+1}(\Omega) \times \widetilde{H}^{s+1}(\Omega) \times\left[H^{s}(\Omega)\right]^{2}, & & s \geq 0,
\end{aligned}
$$

together with their respective dual spaces

$$
\begin{aligned}
X_{s}^{*} & =H^{-(s+1)}(\Omega) \times \widetilde{H}^{-(s+1)}(\Omega) \times\left[H^{-(s+2)}(\Omega)\right]^{2}, & & s \geq 0, \\
Y_{s}^{*} & =H^{-(s+1)}(\Omega) \times \widetilde{H}^{-(s+1)}(\Omega) \times\left[H^{-s}(\Omega)\right]^{2}, & & s \geq 0 .
\end{aligned}
$$


For $U \in D$ the estimate (20) holds for all $q \geq 0$ and can be written as

$$
\|U\|_{X_{q}} \leq\|\mathscr{L} U\|_{Y_{q}} \text {. }
$$

The operator $\mathscr{L}: X_{s} \mapsto Y_{s}$ together with (4) defines a selfadjoint boundary value problem, and therefore the estimate (20) will also hold for the solutions of the adjoint boundary value problem. We shall prove that

$$
\|U\|_{Y_{s}^{*}} \leq\|\mathscr{L} U\|_{X_{s}^{*}} \quad \forall U \in D, s \geq 0 .
$$

By the definition of the dual norm, uniqueness of the solutions to (2)-(4), (7) and by $(20)$,

$$
\begin{aligned}
\|U\|_{Y_{s}^{*}} & =\sup _{H \in D ; H \neq 0} \frac{(U, H)}{\|H\|_{Y_{s}}}=\sup _{V \in D ; V \neq 0} \frac{(U, \mathscr{L} V)}{\|\mathscr{L} V\|_{Y_{s}}} \\
& \leq \sup _{V \in D ; V \neq 0} \frac{(\mathscr{L} U, V)}{\|V\|_{X_{s}}}=\|\mathscr{L} U\|_{X_{s}^{*}} .
\end{aligned}
$$

This establishes (20) for $q \leq-2$ and $q \geq 0$. For the intermediate values of $q$ the result follows by interpolation.

\section{THE STANDARD LEAST SQUARES FUNCTIONAL}

We now consider the finite element approximation of solutions of the velocityvorticity-pressure formulation of the Stokes equations based on the minimization of a least squares functional that is defined in terms of the norms indicated by the ADN theory. We shall first address the general boundary condition (3), and then specialize the results to the specific boundary conditions (4) and (5). As we shall see, for the boundary condition (5), this results in a practical method having optimal accuracy. However, for the boundary condition (4), although conforming approximations are again optimally accurate, they require the use of continuously differentiable finite element functions, and therefore are not very practical.

Let us assume that $\left\{s_{i}\right\}=\left\{s_{1}, s_{2}, s_{3}, s_{4}\right\}$ and $\left\{t_{j}\right\}=\left\{t_{1}, t_{2}, t_{3}, t_{4}\right\}$ are indices for which the boundary operator (3) satisfies the Complementing Condition and that the problem (2)-(3) has a unique solution. (The latter assumption may require additional information of the type (7).) Then, we define the standard least squares functional for the problem (2)-(3) by

$$
\begin{aligned}
\mathscr{J}(U)= & \left\|\operatorname{curl} \omega+\operatorname{grad} p-\mathbf{f}_{1}\right\|_{\left(-s_{1},-s_{2}\right)}^{2}+\left\|\operatorname{curl} \mathbf{u}-\omega-f_{2}\right\|_{-s_{3}}^{2} \\
& +\left\|\operatorname{div} \mathbf{u}-f_{3}\right\|_{-s_{4}}^{2} .
\end{aligned}
$$

Recall that $\operatorname{deg} \mathscr{L}_{i j} \leq s_{i}+t_{j}$; hence, minimization of (21) is meaningful over a suitable subspace $\mathrm{U}$ of $H^{t_{1}}(\Omega) \times H^{t_{2}}(\Omega) \times H^{t_{3}}(\Omega) \times H^{t_{4}}(\Omega)$. The subspace $\mathbf{U}$ will depend on the particular boundary operator, i.e., it will be defined by requiring that homogeneous boundary conditions are satisfied. The least squares principle is then given by

$$
\text { seek } U=(\omega, p, \mathbf{u}) \in \mathbf{U} \text { such that } \mathscr{J}(U) \leq \mathscr{J}(\widehat{U}) \forall \widehat{U}=(\hat{\omega}, \hat{p}, \hat{\mathbf{u}}) \in \mathbf{U} .
$$

Standard techniques of the calculus of variations may be used to deduce that any solution $U$ of (22) necessarily satisfies the variational problem

$$
\text { find } U \in \mathbf{U} \text { such that } \mathscr{B}(U, V)=\mathscr{F}(V) \forall V \in \mathbf{U} \text {, }
$$


where, for $U=(\omega, p, \mathbf{u})$ and $V=(\phi, q, \mathbf{v})$, (24)

$$
\begin{gathered}
\mathscr{B}(U, V)=(\operatorname{curl} \omega+\operatorname{grad} p, \operatorname{curl} \phi+\operatorname{grad} q)_{\left(-s_{1},-s_{2}\right)} \\
+(\operatorname{curl} \mathbf{u}-\omega, \operatorname{curl} \mathbf{v}-\phi)_{-s_{3}}+(\operatorname{div} \mathbf{u}, \operatorname{div} \mathbf{v})_{-s_{4}}, \\
\mathscr{F}(V)=\left(\operatorname{curl} \phi+\operatorname{grad} q, \mathbf{f}_{1}\right)_{\left(-s_{1},-s_{2}\right)}+\left(\operatorname{curl} \mathbf{v}-\phi, f_{2}\right)_{-s_{3}}+\left(\operatorname{div} \mathbf{v}, f_{3}\right)_{-s_{4}} .
\end{gathered}
$$

As a result of the a priori estimates (13) and the fact that we have assumed that (2)-(3) have at most one solution, one can easily establish the existence and uniqueness of the solution of the variational problem (23).

Proposition 2. The problem (23) has a unique solution $U \in \mathbf{U}$. This solution is the unique minimizer of the functional (21).

Proof. Using (13) with $q=0$, the fact that (2)-(3) have at most one solution, and the fact that the order of $\mathscr{L}_{i j}$ is bounded by $s_{i}+t_{j}$, we find that

$$
\begin{aligned}
& C_{1}\left(\|\omega\|_{t_{1}}^{2}+\|p\|_{t_{2}}^{2}+\left\|\mathbf{u}_{1}\right\|_{t_{3}}^{2}+\left\|\mathbf{u}_{2}\right\|_{t_{4}}^{2}\right) \\
& \quad \leq\|\operatorname{curl} \omega+\operatorname{grad} p\|_{-s_{1},-s_{2}}^{2}+\|\operatorname{curl} \mathbf{u}-\omega\|_{-s_{3}}^{2}+\|\operatorname{div} \mathbf{u}\|_{-s_{4}}^{2} \\
& \quad=\mathscr{B}(U, U),
\end{aligned}
$$

i.e., the form $\mathscr{B}(\cdot, \cdot)$ is coercive on the space $\mathbf{U} \times \mathbf{U}$. Continuity of the form is trivial and thus, by the Lax-Milgram lemma, the problem (23) has a unique solution $U \in \mathbf{U}$. By the definition of (23), this solution will also be the unique minimizer of the least squares functional (21).

3.1. Discretization of the standard least squares principle. For the conforming discretizations of the standard least squares principle, we shall need finitedimensional subspaces $S_{j}$ of $H^{t_{j}}(\Omega)$. These spaces are parametrized by a parameter $h$; for example, $h$ is usually some measure of the grid size; the grid itself need not be uniform. We assume the following approximation property of the spaces $S_{j}$ : there exists a $d \geq 0$ such that for every $u \in H^{d+t_{j}}(\Omega)$ there exists an element $v^{h} \in S_{j}$ such that for $0 \leq r \leq t_{j}$

$$
\left\|u-v^{h}\right\|_{r} \leq C h^{d+t_{j}-r}\|u\|_{d+t_{j}} .
$$

Let $\mathbf{U}^{h}=S_{1} \times S_{2} \times S_{3} \times S_{4}$; then the discretization of (22) is given by

$$
\begin{aligned}
& \text { seek } U^{h}=\left(\omega^{h}, p^{h}, \mathbf{u}^{h}\right) \in \mathbf{U}^{h} \\
& \text { such that } \mathscr{J}\left(U_{h}\right) \leq \mathscr{J}\left(\widehat{U}^{h}\right) \forall \widehat{U}_{h}=\left(\hat{\omega}^{h}, \hat{p}^{h}, \hat{\mathbf{u}}^{h}\right) \in \mathbf{U}^{h} .
\end{aligned}
$$

One easily sees that $(27)$ is equivalent to the variational problem:

$$
\text { find } U^{h} \in \mathbf{U}^{h} \quad \text { such that } \mathscr{B}\left(U^{h}, V^{h}\right)=\mathscr{F}\left(V^{h}\right) \forall V^{h} \in \mathbf{U}^{h} \text {; }
$$

clearly (28) is a discrete version of (23). By assumption, $\mathbf{U}^{h}$ is a subspace of $\mathbf{U}$; hence, the inequality (25) holds for all functions $U^{h} \in \mathbf{U}^{h}$. Thus, the discrete problem (28) is coercive, has a unique solution $U^{h}$, and this solution is the unique minimizer for the problem (27). We note that (28) corresponds to a linear system of algebraic equations with a symmetric, positive definite coefficient matrix. An estimate of the error $U-U^{h}$ can now be deduced in a completely standard manner. 
Theorem 3. Let $U=(\omega, p, \mathbf{u}) \in \mathbf{U}$ and $U^{h}=\left(\omega^{h}, p^{h}, \mathbf{u}^{h}\right) \in \mathbf{U}^{h}$ be the solutions of the problems (23) and (28), respectively. Assume that $S_{j}, j=$ $1,2,3,4$, satisfy the approximation property (26). Assume that for some $q \geq$ $0, \omega \in H^{q+t_{1}}(\Omega), p \in H^{q+t_{2}}(\Omega)$, and $\mathbf{u} \in H^{q+t_{3}}(\Omega) \times H^{q+t_{4}}(\Omega)$. Let $\tilde{q}=$ $\min \{d, q\}$. Then,

$$
\begin{aligned}
& \left\|\omega-\omega^{h}\right\|_{t_{1}}+\left\|p-p^{h}\right\|_{t_{2}}+\left\|\mathbf{u}-\mathbf{u}^{h}\right\|_{\left(t_{3}, t_{4}\right)} \\
& \leq C h^{\tilde{q}}\left(\|\omega\|_{\tilde{q}+t_{1}}+\|p\|_{\tilde{q}+t_{2}}+\|\mathbf{u}\|_{\left(\tilde{q}+t_{3}, \tilde{q}+t_{4}\right)}\right) .
\end{aligned}
$$

Proof. Let $\|U\|^{2}=\|\omega\|_{t_{1}}^{2}+\|p\|_{t_{2}}^{2}+\|\mathbf{u}\|_{t_{3}, t_{4}}^{2}$. Using the orthogonality relation $\mathscr{B}\left(U-U^{h}, V^{h}\right)=0 \forall V^{h} \in \mathbf{U}^{h}$ and the inequality (25), we find that

$$
\begin{aligned}
C_{1}\left\|U-U^{h}\right\|^{2} & \leq \mathscr{B}\left(U-U^{h}, U-U^{h}\right) \\
& =\mathscr{B}\left(U-U^{h}, U-V^{h}\right)+\mathscr{B}\left(U-U^{h}, V^{h}-U^{h}\right) \\
& =\mathscr{B}\left(U-U^{h}, U-V^{h}\right) \leq C_{2}\left\|U-U^{h}\right\|\left\|U-V^{h}\right\| .
\end{aligned}
$$

Above, $V^{h}$ is an arbitrary element of $\mathbf{U}^{h}$; hence,

$$
\left\|U-U^{h}\right\| \leq C_{3} \inf _{V^{h} \in \mathbf{U}^{h}}\left\|U-V^{h}\right\| .
$$

Now, (26) and $\tilde{q}=\min \{d, q\}$ implies that we can find $V_{h}=\left(\phi^{h}, q^{h}, \mathbf{v}^{h}\right) \in \mathbf{U}^{h}$ such that

$$
\begin{aligned}
\left\|U-V^{h}\right\| & =\left(\left\|w-\phi^{h}\right\|_{t_{1}}^{2}+\left\|p-q^{h}\right\|_{t_{2}}^{2}+\left\|\mathbf{u}-\mathbf{v}^{h}\right\|_{\left(t_{3}, t_{4}\right)}^{2}\right)^{1 / 2} \\
& \leq h^{\tilde{q}}\left(\|w\|_{\tilde{q}+t_{1}}+\|p\|_{\tilde{q}+t_{2}}+\|\mathbf{u}\|_{\left(\tilde{q}+t_{3}, \tilde{q}+t_{4}\right)}\right),
\end{aligned}
$$

which completes the proof of the theorem.

The estimate (29) is optimal in the sense that if a component of the solution belongs to $H^{\tilde{q}+t_{j}}(\Omega)$, then $h^{\tilde{q}}$ is the best possible rate of convergence for the error measured in the $H^{t_{j}}(\Omega)$-norm.

3.1.1. Pressure-normal velocity boundary conditions. Let us now specialize the above results to the homogeneous boundary condition (5). According to $\S 2.1 .1$, we can choose the indices $s_{i}=0$ and $t_{j}=1$. Then, the least squares functional (21) involves only $L^{2}(\Omega)$-norms of the residuals of all the equations, i.e., we have that

$$
\mathscr{J}(U)=\left\|\operatorname{curl} \omega+\operatorname{grad} p-\mathbf{f}_{1}\right\|_{0}^{2}+\left\|\operatorname{curl} \mathbf{u}-\omega-f_{2}\right\|_{0}^{2}+\left\|\operatorname{div} \mathbf{u}-f_{3}\right\|_{0}^{2}
$$

and $\mathbf{U}=H^{1}(\Omega) \times H_{0}^{1}(\Omega) \times \mathbf{H}_{n}^{1}(\Omega)$, where $\mathbf{H}_{n}^{1}(\Omega)$ denotes the subspace of $H^{1}(\Omega) \times H^{1}(\Omega)$ whose members have normal components equal to zero on the boundary. Furthermore, for the conforming discretization of (23) one can employ finite-dimensional subspaces $S_{j}$ of $H^{1}(\Omega)$. For example, we can use piecewise linear elements or, in general, any $C^{0}$ piecewise polynomial finite element space. For the sake of concreteness, let us choose piecewise quadratic finite element spaces. It is well known (see [13]) that for every function $u$ in $H^{3}(\Omega)$ there exists a finite element function $v^{h}$ such that $\left\|u-v^{h}\right\|_{r} \leq$ $C h^{3-r}\|\dot{u}\|_{3}$ for $r=0$ or 1 . Hence, if we choose $d=2$ in (26) and assume that $q=2$ in Theorem 3, then (29) yields that the error estimate for piecewise quadratic approximations is given by

$$
\left\|\omega-\omega^{h}\right\|_{1}+\left\|p-p^{h}\right\|_{1}+\left\|\mathbf{u}-\mathbf{u}^{h}\right\|_{1} \leq C h^{2}\left(\|w\|_{3}+\|p\|_{3}+\|\mathbf{u}\|_{3}\right) .
$$

Clearly, this estimate is optimal for quadratic finite element spaces. 
3.1.2. Velocity boundary conditions. Next we consider the case when (2) is supplemented with the homogeneous velocity boundary condition (4). According to $\S 2.1 .1$, now $s_{3}=s_{4}=-1$ and $t_{3}=t_{4}=2$; we still have that $s_{1}=s_{2}=0$ and $t_{1}=t_{2}=1$. Thus, the standard least squares functional (21) now involves the $H^{1}(\Omega)$-norms of the residuals of some of the equations, i.e., we have that

$$
\mathcal{J}(U)=\left\|\operatorname{curl} \omega+\operatorname{grad} p-\mathbf{f}_{1}\right\|_{0}^{2}+\left\|\operatorname{curl} \mathbf{u}-\omega-f_{2}\right\|_{1}^{2}+\left\|\operatorname{div} \mathbf{u}-f_{3}\right\|_{1}^{2}
$$

and $\mathbf{U}=H^{1}(\Omega) \times \widetilde{H}^{1}(\Omega) \times\left(H^{2}(\Omega) \cap H_{0}^{1}(\Omega)\right)^{2}$. This implies that for a conforming discretization, the velocity field should be approximated in finite-dimensional subspaces of $H^{2}(\Omega)$. Hence, the straightforward conforming discretization of (23) in this case bears no direct advantage over discretizations of least squares principles based on the primitive variable Stokes equations (see [2]). We can conclude that from a practical point of view the standard least squares functional is appropriate only for those problems where the Complementing Condition holds under the assumption of the equal order of differentiability.

\section{THE MESH-DEPENDENT LEAST SQUARES PRINCIPLE}

In the previous section we saw that the application of the standard least squares method to the velocity-vorticity-pressure form of the Stokes equations with velocity boundary conditions resulted in approximate methods that required the use of continuously differentiable finite element functions. We now examine the possibility of devising a least squares method that allows the use of merely continuous finite element functions. Of course, for the velocity boundary conditions we cannot use the functional (30) instead of (32). The reason for this is that (30) was defined using indices $s_{i}$ and $t_{j}$ for which the Complementing Condition does not hold with (4) and the ADN theory does not apply. In particular, and notwithstanding previous claims made in the literature, the inequality

$$
\|\operatorname{curl} \omega+\operatorname{grad} p\|_{0}^{2}+\|\operatorname{curl} \mathbf{u}-\omega\|_{0}^{2}+\|\operatorname{div} \mathbf{u}\|_{0}^{2} \geq C\left(\|\omega\|_{1}^{2}+\|p\|_{1}^{2}+\|\mathbf{u}\|_{1}^{2}\right)
$$

does not hold for velocity boundary conditions. For example, let $\mathbf{u}=\mathbf{0}$ and let $\omega$ and $p$ be conjugate harmonic functions. Then,

$$
\|\operatorname{curl} \omega+\operatorname{grad} p\|_{0}^{2}+\|\operatorname{curl} \mathbf{u}-\omega\|_{0}^{2}+\|\operatorname{div} \mathbf{u}\|_{0}^{2}=\|\omega\|_{0}^{2} .
$$

However, for harmonic functions it is not true, in general, that $\|\omega\|_{0} \geq C\|\omega\|_{1}$. (See also Remark 1 of $\S 2.1 .1$.)

Thus, in order to take full advantage of the velocity-vorticity-pressure equations, we shall consider a mesh-dependent functional which will involve only weighted $L^{2}$-norms of the residuals. The choice of the mesh-dependent weights is dictated by the "inverse inequalities" which hold for a wide range of finite element spaces $S^{h}$; see [13]. Specifically, we have that for $v^{h} \in S^{h} \subset H^{m}(\Omega)$ and $0 \leq r \leq m$

$$
\left\|v^{h}\right\|_{r} \leq C h^{-r}\left\|v^{h}\right\|_{0},
$$

i.e., $h^{s_{i}}\left\|v^{h}\right\|_{0}$ can "simulate" $\left\|v^{h}\right\|_{-s_{i}}$. Hence, the $H^{\left(-s_{i}\right)}$-norm of the residual of the $i$ th equation which appears in the standard functional (21) can be replaced by the $L^{2}$-norm of the same residual multiplied by $h^{2 s_{i}}$. 
After all norms in the standard least squares functional are replaced with properly weighted $L^{2}$-norms, we obtain the mesh-dependent (or weighted) least squares functional

$$
\begin{aligned}
\mathscr{J}^{h}(U)= & h^{2 s_{1}}\left\|\omega_{y}+p_{x}-f_{11}\right\|_{0}^{2}+h^{2 s_{2}}\left\|p_{y}-\omega_{x}-f_{12}\right\|_{0}^{2} \\
& +h^{2 s_{3}}\left\|\operatorname{curl} \mathbf{u}-\omega-f_{2}\right\|_{0}^{2}+h^{2 s_{4}}\left\|\operatorname{div} \mathbf{u}-f_{3}\right\|_{0}^{2} .
\end{aligned}
$$

If all equation indices are equal, one has the common and unimportant factor $h^{2 s_{i}}$ and, insofar as minimization is concerned, the functional (34) is identical to the standard one $(21)$.

Using standard techniques of the calculus of variations, one can show, for any fixed value of $h$, that minimization of (34) over an appropriate space $\mathbf{U}$ is equivalent to the variational problem

$$
\text { find } U \in \mathbf{U} \text { such that } \mathscr{B}^{h}(U, V)=\mathscr{F}^{h}(V) \forall V \in \mathbf{U} \text {, }
$$

where $U=(\omega, p, \mathbf{u}), V=(\phi, q, \mathbf{v})$,

$$
\begin{aligned}
\mathscr{B}^{h}(U, V)= & \int_{\Omega}\left(h^{2 s_{1}}\left(\omega_{y}+p_{x}\right)\left(\phi_{x}+q_{y}\right)+h^{2 s_{2}}\left(-\omega_{x}+p_{y}\right)\left(-\phi_{x}+q_{y}\right)\right) d x \\
& +\int_{\Omega}\left(h^{2 s_{3}}(\operatorname{curl} \mathbf{u}-\omega)(\operatorname{curl} \mathbf{v}-\phi)+h^{2 s_{4}}(\operatorname{div} \mathbf{u})(\operatorname{div} \mathbf{v})\right) d x, \\
\mathscr{F}^{h}(V)= & \int_{\Omega}\left(h^{2 s_{1}}\left(\phi_{x}+q_{y}\right) f_{11}+h^{2 s_{2}}\left(-\phi_{x}+q_{y}\right) f_{12}\right. \\
& \left.+h^{2 s_{3}}(\operatorname{curl} \mathbf{v}-\phi) f_{2}+h^{2 s_{4}}(\operatorname{div} \mathbf{v}) f_{3}\right) d x .
\end{aligned}
$$

For the discretization of (35) we consider a finite-dimensional subspace $\mathbf{U}^{h}$ of $\mathbf{U}$ and pose the problem:

$$
\text { find } U^{h} \in \mathbf{U}^{h} \quad \text { such that } \mathscr{B}^{h}\left(U^{h}, V^{h}\right)=\mathscr{F}\left(V^{h}\right) \forall V^{h} \in \mathbf{U}^{h} \text {, }
$$

where $U^{h}=\left(\omega^{h}, p^{h}, \mathbf{u}^{h}\right)$ and $V^{h}=\left(\phi^{h}, q^{h}, \mathbf{v}^{h}\right)$. Evidently, $U^{h}$ is the minimizer of $\mathscr{J}^{h}(U)$ over $\mathbf{U}^{h}$.

4.1. Error estimates. The error analysis for the approximations $U^{h}$ generated by the variational problem (37) is significantly more elaborate than the error analysis for the standard least squares method of $\S 3.1$. In the earlier case, coercivity of the form $\mathscr{B}(\cdot, \cdot)$ stems directly from the ADN a priori inequality (13). For the form (36), such a conclusion cannot be drawn immediately, and proof of the stability of (36) requires the use of $(20)$ with $q<0$. In this regard we follow some ideas of [2].

Here, we consider homogeneous velocity boundary conditions; for more general treatments as well as for other boundary conditions, we refer to the methods given in [2] and [4]. With the appropriate indices $s_{1}=s_{2}=0, s_{3}=s_{4}=-1$, $t_{1}=t_{2}=1$, and $t_{3}=t_{4}=2$ for this boundary condition, the weighted least squares functional (34) becomes

$$
\begin{aligned}
\mathscr{J}^{h}(U)= & \left\|\omega_{y}+p_{x}-f_{11}\right\|_{0}^{2}+\left\|-\omega_{x}+p_{y}-f_{12}\right\|_{0}^{2} \\
& +h^{-2}\left\|\operatorname{curl} \mathbf{u}-\omega-f_{2}\right\|_{0}^{2}+h^{-2}\left\|\operatorname{div} \mathbf{u}-f_{3}\right\|_{0}^{2} .
\end{aligned}
$$

By $\mathscr{L}_{i j}$ we shall denote the differential operators of the system (2); for example, $\mathscr{L}_{21}=-\partial / \partial x$ and $\mathscr{L}_{44}=\partial / \partial y$. The orders of each $\mathscr{L}_{i j}$ are, of course, 
bounded by $s_{i}+t_{j}$. We let $U_{j}$ and $U_{j}^{h}$ denote the $j$ th component of the solution of $U=(\omega, p, \mathbf{u})$ and its weighted least squares approximation $U^{h}=$ $\left(\omega^{h}, p^{h}, \mathbf{u}^{h}\right)$, respectively.

We shall assume that the solution $(\omega, p, \mathbf{u})$ of (2) and (4) (with $\mathbf{u}^{0}=\mathbf{0}$ ) satisfies

$$
(\omega, p, \mathbf{u}) \in \mathbf{U}=H^{d+1}(\Omega) \times \tilde{H}^{d+1} \times\left[H^{d+2}(\Omega) \cap H_{0}^{1}(\Omega)\right]^{2} .
$$

In addition, we require that $d$ be subject to the condition

$$
\max _{i=1, \ldots, 4}\left(2 s_{i}-d\right) \leq \min _{i=1, \ldots, 4} s_{i}
$$

For velocity boundary conditions we have that $\min _{i=1, \ldots, 4} s_{i}=-1$ and $\max _{i=1, \ldots, 4} 2 s_{i}=0$ and hence, (40) requires that $d \geq 1$. The necessity of this restriction will become clear in the proof of Proposition 4 below.

In contrast to the standard least squares method (32) for velocity boundary conditions, we can now choose to approximate each unknown in a subspace of $H^{1}$. Therefore, we consider minimization of (38) over the following finitedimensional space:

$$
\left(w^{h}, p^{h}, \mathbf{u}^{h}\right)=\mathbf{U}^{h}=S_{1} \times S_{2} \times S_{3} \times S_{4} \subset H^{1}(\Omega) \times \widetilde{H}^{1}(\Omega) \times\left[H_{0}^{1}(\Omega)\right]^{2} .
$$

Each space $S_{j}$ in (41) will be required to approximate optimally with respect to the corresponding function space in (39), i.e., the inequality (26) must hold with the appropriate values of $t_{j}$. Note that the required approximation properties of the spaces $S_{j}$ do not imply higher smoothness properties of these spaces because the latter solely depend on the highest order of differentiation in the weighted least squares functional (38).

We begin with a continuity-type of estimate for the solutions of the discrete variational problem (37).

Proposition 3. Let $U=(\omega, p, \mathbf{u}) \in \mathbf{U}$ be arbitrary functions, let $\mathbf{f}_{1}, f_{2}$, and $f_{3}$ be defined by (2), and let $U^{h}=\left(\omega^{h}, p^{h}, \mathbf{u}^{h}\right) \in \mathbf{U}^{h}$ be the corresponding least squares approximation given by (37). Then

$$
\mathscr{B}^{h}\left(U-U^{h}, U-U^{h}\right)^{1 / 2} \leq h^{d}\left(\|\omega\|_{d+1}+\|p\|_{d+1}+\|\mathbf{u}\|_{d+2}\right) .
$$

Proof. The error $U-U^{h}$ satisfies the orthogonality relation $\mathscr{B}^{h}\left(U-U^{h}, W^{h}\right)=$ 0 , and therefore

$$
\mathscr{B}^{h}\left(U-U^{h}, U-U^{h}\right)^{1 / 2} \leq \mathscr{B}^{h}\left(U-W^{h}, U-W^{h}\right)^{1 / 2} \quad \forall W^{h} \in \mathbf{U}^{h} .
$$

Let $W^{h}=\left(\phi^{h}, q^{h}, \mathbf{v}^{h}\right)$; using the approximation properties of the spaces $S_{j}$, we can further estimate $\mathscr{B}^{h}\left(U-W^{h}, U-W^{h}\right)$ as follows:

$$
\begin{aligned}
\mathscr{B}^{h} & \left(U-W^{h}, U-W^{h}\right)^{1 / 2} \\
& =\left(\left\|\operatorname{curl}\left(\omega-\phi^{h}\right)-\operatorname{grad}\left(p-q^{h}\right)\right\|_{0}^{2}+h^{-2}\left\|\operatorname{curl}\left(\mathbf{u}-\mathbf{v}^{h}\right)-\left(\omega-\phi^{h}\right)\right\|_{0}^{2}+h^{-2}\left\|\operatorname{div}\left(\mathbf{u}-\mathbf{v}^{h}\right)\right\|_{0}^{2}\right)^{1 / 2} \\
& \leq C\left(\left\|\omega-\phi^{h}\right\|_{1}+h^{-1}\left\|\omega-\phi^{h}\right\|_{0}+\left\|p-q^{h}\right\|_{1}+h^{-1}\left\|\mathbf{u}-\mathbf{v}^{h}\right\|_{1}\right) \\
& \leq C h^{d}\left(\|\omega\|_{d+1}+\|p\|_{d+1}+\|\mathbf{u}\|_{d+2}\right) . \quad \square
\end{aligned}
$$

The next proposition establishes the stability of the form $\mathscr{B}(\cdot, \cdot)$.

Proposition 4. Let the spaces $S_{j}$ be defined by (41) with $d$ satisfying (40). Let $q$ be a nonpositive number such that

$$
\max _{i}\left(2 s_{i}-d\right) \leq q \leq \min _{i} s_{i} .
$$


Then, for $U$ and $U^{h}$ as in Proposition 3,

(44) $\left\|\omega-\omega^{h}\right\|_{q+1}+\left\|p-p^{h}\right\|_{q+1}+\left\|\mathbf{u}-\mathbf{u}^{h}\right\|_{q+2} \leq C h^{-q} \mathscr{B}^{h}\left(U-U^{h}, U-U^{h}\right)^{1 / 2}$.

We postpone the proof of Proposition 4 until the end of this section. The final error estimate now easily follows.

Theorem 4. Let $U \in \mathbf{U}$ solve the problem (2)-(4), (7), and let $q$ and $d$ be defined as in Proposition 4. Then, the weighted least squares solution $U^{h} \in \mathbf{U}^{h}$ satisfies

(45) $\left\|\omega-\omega^{h}\right\|_{q+1}+\left\|p-p^{h}\right\|_{q+1}+\left\|\mathbf{u}-\mathbf{u}^{h}\right\|_{q+2} \leq C h^{d-q}\left(\|\omega\|_{d+1}+\|p\|_{d+1}+\|\mathbf{u}\|_{d+2}\right)$.

Proof. By (42) and (44) it follows that

$$
\begin{aligned}
\| \omega- & \omega^{h}\left\|_{q+1}+\right\| p-p^{h}\left\|_{q+1}+\right\| \mathbf{u}-\mathbf{u}^{h} \|_{q+2} \leq C h^{-q} \mathscr{B}^{h}\left(U-U^{h}, U-U^{h}\right)^{1 / 2} \\
& \leq h^{-q} \inf _{W_{h} \in \mathbf{U}_{h}} \mathscr{B}^{h}\left(U-W^{h}, U-W^{h}\right)^{1 / 2} \\
& \leq C h^{d-q}\left(\|\omega\|_{d+1}+\|p\|_{d+1}+\|\mathbf{u}\|_{d+2}\right) .
\end{aligned}
$$

In Theorem 4 we must assume $q \leq-1$, i.e., (45) gives only $L^{2}$-norm estimates for the error in the vorticity and the pressure approximations. If the inverse inequality (33) holds for the spaces $S_{j}$, one can obtain stronger $H^{1}$ norm estimates for these errors.

Corollary 1. Suppose the hypotheses of Theorem 4 hold and that the inverse inequality (33) holds for the spaces $S_{1}$ and $S_{2}$. Then,

$$
\begin{aligned}
\left\|\omega-\omega^{h}\right\|_{1, \Omega} & \leq C h^{d}\left(\|\omega\|_{d+1}+\|p\|_{d+1}+\|\mathbf{u}\|_{d+2}\right), \\
\left\|p-p^{h}\right\|_{1, \Omega} & \leq C h^{d}\left(\|\omega\|_{d+1}+\|p\|_{d+1}+\|\mathbf{u}\|_{d+2}\right) .
\end{aligned}
$$

Proof. Using the approximation properties (26) and the estimate (45) with $q=$ -1 , we find that

$$
\begin{aligned}
\left\|\omega-\omega^{h}\right\|_{1, \Omega} & \leq\left\|\omega-\phi^{h}\right\|_{1, \Omega}+\left\|\omega^{h}-\phi^{h}\right\|_{1, \Omega} \\
& \leq C\left(h^{d}\|\omega\|_{d+1}+h^{-1}\left\|\omega^{h}-\phi^{h}\right\|_{0, \Omega}\right) \\
& \leq C\left(h^{d}\|\omega\|_{d+1}+h^{-1}\left(\left\|\omega-\phi^{h}\right\|_{0, \Omega}+\left\|\omega-\omega^{h}\right\|_{0, \Omega}\right)\right) \\
& \leq C\left(h^{d}\|\omega\|_{d+1}+h^{d-q-1}\left(\|\omega\|_{d+1}+\|p\|_{d+1}+\|\mathbf{u}\|_{d+2}\right)\right) \\
& \leq C h^{d}\left(\|\omega\|_{d+1}+\|p\|_{d+1}+\|\mathbf{u}\|_{d+2}\right) .
\end{aligned}
$$

The estimate (47) is derived in an identical manner.

Let us now prove Proposition 4. Here we follow the ideas of [2].

Proof of Proposition 4. We apply (20) with $q \leq-1$ to the error $U-U^{h}$ to find that

$$
\begin{aligned}
C_{1}\left(\left\|\omega-\omega^{h}\right\|_{q+1}^{2}+\left\|p-p^{h}\right\|_{q+1}^{2}+\left\|\mathbf{u}-\mathbf{u}^{h}\right\|_{q+2}^{2}\right) \\
\leq \\
\quad\left\|\operatorname{curl}\left(\omega-\omega^{h}\right)+\operatorname{grad}\left(p-p^{h}\right)\right\|_{q}^{2}+\left\|\operatorname{curl}\left(\mathbf{u}-\mathbf{u}^{h}\right)-\left(\omega-\omega^{h}\right)\right\|_{q+1}^{2} \\
\quad+\left\|\operatorname{div}\left(\mathbf{u}-\mathbf{u}^{h}\right)\right\|_{q+1}^{2} .
\end{aligned}
$$

The terms on the right-hand side above are of the form $\left\|\sum_{j} \mathscr{L}_{i j}\left(U_{j}-U_{j}^{h}\right)\right\|_{q-s_{i}}$ and thus the estimate (44) will follow if each one of these terms can be estimated 
by $\mathscr{B}^{h}\left(U-U^{h}, U-U^{h}\right)$. This can be accomplished by interpolation between the spaces $H^{s_{i}-d}(\Omega)$ and $L^{2}(\Omega)$. By definition (9),

$$
\left\|\sum_{j} \mathscr{L}_{i j}\left(U_{j}-U_{j}^{h}\right)\right\|_{s_{i}-d}=\sup _{f_{i} \in \mathscr{D}} \frac{\left(\sum_{j} \mathscr{L}_{i j}\left(U_{j}-U_{j}^{h}\right), f_{i}\right)}{\left\|f_{i}\right\|_{d-s_{i}}},
$$

where $\mathscr{D}$ is a space of smooth functions which is dense in $H^{d-s_{i}}(\Omega)$ or $\widetilde{H}^{d-s_{i}}(\Omega)$. The duality pairing which appears above is also meaningful as an $L^{2}$ integral. Let $\mathbf{f}_{1} \in \mathscr{D}(\bar{\Omega})^{2}, f_{2} \in \mathscr{D}(\bar{\Omega})$, and $f_{3} \in \widetilde{\mathscr{D}}(\bar{\Omega})$. We can choose this space for $f_{3}$ because of our earlier assumption that the boundary conditions are satisfied exactly. In this case, $\sum_{j} \mathscr{L}_{4 j}\left(U_{j}-U_{j}^{h}\right)=\operatorname{div}\left(\mathbf{u}-\mathbf{u}^{h}\right)$ has zero mean, and the supremum in the corresponding dual norm has to be taken with respect to $\widetilde{\mathscr{D}}(\bar{\Omega})$. Therefore, $f_{3}$ meets the compatibility condition (7) for the Stokes problem (2)-(4), and the system

$$
\begin{aligned}
\operatorname{curl} \phi+\operatorname{grad} q & =\mathbf{f}_{1} & & \text { in } \Omega, \\
\operatorname{curl} \mathbf{v}-\phi & =f_{2} & & \text { in } \Omega, \\
\operatorname{div} \mathbf{v} & =f_{3} & & \text { in } \Omega, \\
\mathbf{v} & =0_{3} & & \text { on } \Gamma
\end{aligned}
$$

will have a unique solution for every smooth right-hand side in the indicated spaces. If the boundary conditions were not exactly satisfied, one would have to consider an arbitrary smooth function $f_{3}$. Then the system (48) must be modified (see $[2,29])$ in order to guarantee its solvability.

Now, let $V=(\phi, q, \mathrm{v})$ be the solution of (48) with only one, say $f_{i}$, nonzero right-hand side, and let $V^{h}$ denote the least squares approximation to $V$ computed by (37). We use orthogonality of the errors, definition (36), and the estimates (42) and (20) to find an upper bound for the term $\left(\sum_{j} \mathscr{L}_{i j}\left(U_{j}-U_{j}^{h}\right), f_{i}\right)$ :

$$
\begin{aligned}
\left(\sum_{j}\right. & \left.\mathscr{L}_{i j}\left(U_{j}-U_{j}^{h}\right), f_{i}\right)=h^{-2 s_{i}}\left(h^{2 s_{i}} \sum_{j} \mathscr{L}_{i j}\left(U_{j}-U_{j}^{h}\right), f_{i}\right) \\
& =h^{-2 s_{i}} \sum_{k}\left(h^{2 s_{k}} \sum_{j} \mathscr{L}_{k j}\left(U_{j}-U_{j}^{h}\right), f_{k}\right) \\
& =h^{-2 s_{i}} \sum_{k}\left(h^{2 s_{k}} \sum_{j} \mathscr{L}_{k j}\left(U_{j}-U_{j}^{h}\right), \sum_{m} \mathscr{L}_{k m} V_{m}\right) \\
& =h^{-2 s_{i}} \mathscr{B}^{h}\left(U-U^{h}, V\right)=h^{-2 s_{i}} \mathscr{B}^{h}\left(U-U^{h}, V-V^{h}\right) \\
& \leq C h^{-2 s_{i}}\left(\mathscr{B}^{h}\left(U-U^{h}, U-U^{h}\right)\right)^{1 / 2}\left(\mathscr{B}{ }^{h}\left(V-V^{h}, V-V^{h}\right)\right)^{1 / 2} \\
& \leq C h^{d-2 s_{i}}\left(\mathscr{B}^{h}\left(U-U^{h}, U-U^{h}\right)\right)^{1 / 2}\left(\|\phi\|_{d+1}+\|q\|_{d+1}+\|\mathbf{v}\|_{d+2}\right) \\
& \leq C h^{d-2 s_{i}}\left(\mathscr{B}^{h}\left(U-U^{h}, U-U^{h}\right)\right)^{1 / 2}\left\|f_{i}\right\|_{d-s_{i}} .
\end{aligned}
$$

Therefore,

$$
\left\|\sum_{j} \mathscr{L}_{i j}\left(U_{j}-U_{j}^{h}\right)\right\|_{s_{i}-d} \leq C h^{d-2 s_{i}}\left(\mathscr{B}^{h}\left(U-U^{h}, U-U^{h}\right)\right)^{1 / 2} .
$$


For the estimate of $\sum_{j} \mathscr{L}_{i j}\left(U_{j}-U_{j}^{h}\right)$ in the norm of $L^{2}(\Omega)$ we use the definition of the form $\mathscr{B}^{h}(\cdot, \cdot)$ to find

$$
\left\|\sum_{j} \mathscr{L}_{i j}\left(U_{j}-U_{j}^{h}\right)\right\|_{0} \leq C h^{-s_{i}}\left(\mathscr{B}^{h}\left(U-U^{h}, U-U^{h}\right)\right)^{1 / 2} .
$$

Now, the estimate for the $\left(q-s_{i}\right)$ th norm can be found by interpolation between $H^{s_{i}-d}(\Omega)$ and $H^{0}(\Omega)$. For $q$ chosen according to (43), one has

$$
s_{i}-d \leq q-s_{i} \leq 0,
$$

and if

$$
\theta=\frac{s_{i}-q}{d-s_{i}}
$$

then the space $H^{s_{i}-q}(\Omega)$ can be defined by interpolation (see [25]):

$$
\left[H^{0}(\Omega), H^{s_{i}-d}(\Omega)\right]_{\theta}=H^{q-s_{i}}(\Omega) .
$$

The application of the interpolation inequality [25] yields

$$
\begin{aligned}
\left\|\sum_{j} \mathscr{L}_{i j}\left(U_{j}-U_{j}^{h}\right)\right\|_{q-s_{i}} & \leq C\left\|\sum_{j} \mathscr{L}_{i j}\left(U_{j}-U_{j}^{h}\right)\right\|_{s_{i}-d}\left\|\sum_{j} \mathscr{L}_{i j}\left(U_{j}-U_{j}^{h}\right)\right\|_{0}^{1-\theta} \\
& \leq C h^{\left(d-2 s_{i}\right) \theta} h^{-s_{i}(1-\theta)}\left(\mathscr{B}^{h}\left(U-U^{h}, U-U^{h}\right)\right)^{1 / 2} \\
& =C h^{-q}\left(\mathscr{B}^{h}\left(U-U^{h}, U-U^{h}\right)\right)^{1 / 2} .
\end{aligned}
$$

Now (44) easily follows.

4.2. Application to some concrete finite element spaces. A few comments are now in order with regard to the error estimates. Let us suppose that $S_{3}$ and $S_{4}$ are chosen to be finite element spaces of continuous piecewise quadratic functions with respect to a given regular (but not necessarily uniform) triangulation. Then, $d=1$ and $q$ must be chosen equal to -1 . For the approximations of the vorticity and the pressure it suffices to consider continuous piecewise linear elements. Then, the estimates (45), (46), and (47) yield

$$
\begin{gathered}
\left\|\omega-\omega^{h}\right\|_{0}+\left\|p-p^{h}\right\|_{0}+\left\|\mathbf{u}-\mathbf{u}^{h}\right\|_{1} \leq C h^{2}\left(\|\omega\|_{2}+\|p\|_{2}+\|\mathbf{u}\|_{3}\right), \\
\left\|\omega-\omega^{h}\right\|_{1} \leq C h\left(\|\omega\|_{2}+\|p\|_{2}+\|\mathbf{u}\|_{3}\right), \\
\left\|p-p^{h}\right\|_{1} \leq C h\left(\|\omega\|_{2}+\|p\|_{2}+\|\mathbf{u}\|_{3}\right)
\end{gathered}
$$

for all solutions of (2) with sufficient regularity. Let us now suppose that $S_{1}$ and $S_{2}$ are also chosen to be piecewise quadratic finite element spaces, so that all fields are approximated with the same discrete spaces defined with respect to the same grid. This will not change the error estimates, and if $\omega$ and $p$ are only in $H^{2}(\Omega)$, then the rates in (50) and (51) are indeed the best one can expect for $H^{2}$ functions, regardless of the approximation spaces used for the pressure and the vorticity. However, if $\omega$ and $p$ are more regular, then the rates in the error estimates (50) and (51) would be optimal only if piecewise linear elements were used for the approximations of the vorticity and the pressure. On the other hand, for smooth $\omega$ and $p$ one can speculate that quadratic elements might improve the convergence rates for the $L^{2}$ - and $H^{1}$-error norms of $\omega$ and $p$ 
to 3 and 2, respectively, despite the fact that this cannot be deduced from our error estimates. In $\S 6$ we present numerical results which suggest that, at least computationally, for smooth solutions $(\omega, p, \mathbf{u})$ convergence rates of the $L^{2}$ and $H^{1}$-norms of the errors for all four fields are indeed 3 and 2 , respectively.

If we use cubic polynomials for the velocity, and quadratic or cubic polynomials for the vorticity and pressure, we have that $d=2$, and then we may choose $q$ such that $-2 \leq q \leq-1$. Then, for sufficiently smooth solutions, by setting $q=-1$ in (45), (46), and (47), we obtain the estimates

$$
\begin{gathered}
\left\|\omega-\omega^{h}\right\|_{0}+\left\|p-p^{h}\right\|_{0}+\left\|\mathbf{u}-\mathbf{u}^{h}\right\|_{1} \leq C h^{3}\left(\|\omega\|_{3}+\|p\|_{3}+\|\mathbf{u}\|_{4}\right), \\
\left\|\omega-\omega^{h}\right\|_{1} \leq C h^{2}\left(\|\omega\|_{3}+\|p\|_{3}+\|\mathbf{u}\|_{4}\right), \\
\left\|p-p^{h}\right\|_{1} \leq C h^{2}\left(\|\omega\|_{3}+\|p\|_{3}+\|\mathbf{u}\|_{4}\right)
\end{gathered}
$$

By setting $q=-2$ in (45), we can also get improved estimates in weaker norms, including the $L^{2}$-norm for the velocity:

$$
\left\|\omega-\omega^{h}\right\|_{-1}+\left\|p-p^{h}\right\|_{-1}+\left\|\mathbf{u}-\mathbf{u}^{h}\right\|_{0} \leq C h^{4}\left(\|\omega\|_{3}+\|p\|_{3}+\|\mathbf{u}\|_{4}\right) .
$$

The requirement that $-d \leq q \leq-1$ implies that our theory does not cover the case of piecewise linear finite element spaces for the velocity.

\section{LEAST SQUARES METHODS FOR THE STOKES EQUATIONS IN 3D}

For practical applications it is important to extend the results of $\S \S 2-4$ to the three-dimensional case. With minor modifications, virtually all results, especially those concerning the error estimates for least squares methods, remain unchanged in three dimensions. Most of the modifications are due to the fact that the velocity-vorticity-pressure formulation of the Stokes equations in three dimensions involves seven unknowns and equations, and thus cannot be elliptic. Once the proper first-order system and boundary conditions are defined, the least squares theory can be easily extended to the three-dimensional case. For example, along the same lines as those developed in $\S 2$, one can show that the Complementing Condition does not hold for the velocity boundary conditions (understood in the context of the velocity-vorticity-pressure equations in 3D) if an equal order of differentiability is assumed for all unknowns. In this section we shall only state the main results concerning the least squares in $3 \mathrm{D}$, and for the details we refer to [4].

The velocity-vorticity-pressure Stokes equations in three dimensions are given by

$$
\begin{array}{rlr}
\operatorname{curl} \omega+\operatorname{grad} p=\mathbf{f} & \text { in } \Omega, \\
\operatorname{curl} \mathbf{u}-\boldsymbol{\omega}=0 & \text { in } \Omega, \\
\operatorname{div} \mathbf{u}=0 & \text { in } \Omega,
\end{array}
$$

where $\Omega$ is an open and bounded set in $\mathbf{R}^{3}$ with smooth boundary $\Gamma$. One has seven unknown scalar fields and seven equations. It is easy to see that the system (52) is not elliptic in the sense of [1]. Hence, following [9] and [21], we add the seemingly redundant relation

$$
\operatorname{div} \boldsymbol{\omega}=0 .
$$

This brings the number of the equations to eight, and for the well-posedness of the system we must add one more unknown, or slack variable; see [10]. Although 
the addition of this variable seemingly changes the differential equations, we shall see that in the end this variable vanishes identically. Thus, we consider the following generalized velocity-vorticity-pressure equations in three dimensions:

$$
\begin{aligned}
\operatorname{curl} \omega+\operatorname{grad} p & =\mathbf{f}_{1} & & \text { in } \Omega, \\
\operatorname{div} \omega & =f_{2} & & \text { in } \Omega, \\
\operatorname{curl} \mathbf{u}+\operatorname{grad} \phi-\omega & =\mathbf{f}_{3} & & \text { in } \Omega, \\
\operatorname{div} \mathbf{u} & =f_{4} & & \text { in } \Omega .
\end{aligned}
$$

One can show that for the differential operator $\mathscr{L}$ in (53), $\operatorname{det} \mathscr{L}(\xi)=|\xi|^{8}$, i.e., (53) is an elliptic system of total order eight, which must be supplemented with four boundary conditions; in contrast, the primitive variable Stokes problem is a system of total order six and needs only three boundary conditions. Of course, in our context, we have the boundary condition on the velocity

$$
\mathbf{u}=\mathbf{u}_{0} \quad \text { on } \Gamma \text {. }
$$

These three boundary conditions suffice for the primitive variable formulation, but the velocity-vorticity-pressure formulation (53) requires one more. It is tempting to choose the fourth boundary condition to be the specification of the normal component of the vorticity on the boundary. Indeed, if $\mathbf{u}=\mathbf{u}_{0}$ on the boundary $\Gamma$, and if we assume that the definition of the vorticity holds all the way to the boundary, at least in the sense that $\omega=$ curl $u$ on $\Gamma$, then we have that

$$
\boldsymbol{\omega} \cdot \mathbf{n}=\mathbf{n} \cdot \operatorname{curl} \mathbf{u}_{0} \quad \text { on } \Gamma,
$$

where $\mathbf{n} \cdot$ curl $_{\mathbf{u}_{0}}$ is computable from $\mathbf{u}_{0}$, i.e., $\mathbf{n} \cdot \operatorname{curl} \mathbf{u}_{0}$ involves only tangential derivatives of the components of $\mathbf{u}_{0}$.

However, we do not need to assume that the differential equation $\boldsymbol{\omega} \cdot \mathbf{n}=$ $\mathbf{n} \cdot$ curl $\mathbf{u}$ holds at the boundary $\Gamma$ if we instead choose for the fourth boundary condition

$$
\phi=0 \quad \text { on } \Gamma,
$$

i.e., a condition on the slack variable $\phi$. Note that if $\mathbf{f}_{3}$ satisfies $\operatorname{div} \mathbf{f}_{3}=0$, as is true for the Stokes system (52), then $\phi$ is a harmonic function, so that, using (55), we have that $\phi=0$ everywhere. (If we instead use (54), we can still conclude that $\phi=$ constant everywhere; in this case, in order to get a unique solution, we have to require that $\phi$ have zero mean.)

Here, we will adopt (55) as the fourth boundary condition. Since, for simplicity, we are considering only homogeneous boundary conditions, the four boundary conditions for the system (53) are given by

$$
\mathbf{u}=\mathbf{0}, \quad \phi=0 \quad \text { on } \Gamma \text {. }
$$

The addition of the seemingly redundant equation $\operatorname{div} \omega=0$ is crucial to the algorithm, as well as to its analysis. However, it is important to point out that the introduction of the slack variable $\phi$ is purely for the purposes of analysis; the least squares algorithm we are about to introduce does not make use of this variable, i.e., it only involves $\omega, p$, and $\mathbf{u}$. In fact, we can carry out the analysis including the slack variable $\phi$, and then specialize all results to the 
case when $\phi=0$. Thus, we obtain results for the system

$$
\begin{aligned}
\operatorname{curl} \boldsymbol{\omega}+\operatorname{grad} p=\mathbf{f}_{1} & \text { in } \Omega, \\
\operatorname{div} \boldsymbol{\omega}=f_{2} & \text { in } \Omega, \\
\operatorname{curl} \mathbf{u}-\boldsymbol{\omega}=\mathbf{f}_{3} & \text { in } \Omega, \\
\operatorname{div} \mathbf{u}=f_{4} & \text { in } \Omega,
\end{aligned}
$$

with boundary condition

$$
\mathbf{u}=\mathbf{0} \quad \text { on } \Gamma
$$

under the assumptions that

$$
\operatorname{div} \mathbf{f}_{3}=0 \quad \text { in } \Omega \text {. }
$$

Of course, because of (56), we also have the compatibility assumption on $f_{4}$ :

$$
\int_{\Omega} f_{4} d x=0,
$$

and in order to get a unique solution, we specify that

$$
\int_{\Omega} p d x=0 \text {. }
$$

Note that the velocity-vorticity-pressure Stokes problem fits into the framework of (57)-(61).

We now summarize some results for the velocity-vorticity-pressure equations which are of central importance for the formulation and analysis of the least squares methods in three dimensions.

Proposition 5. Let $U=(\omega, p, \mathbf{u})$. Then

- For every solution $(\mathbf{u}, p)$ of the primitive variable Stokes problem, $(\omega=$ curl $\mathbf{u}, p, \mathbf{u}$ ) is a solution of (57) and (58) with $f_{2}=0, \mathbf{f}_{3}=0$, and $f_{4}=0$, and for every solution $U$ of the latter, $(\mathbf{u}, p)$ solves the Stokes problem.

- The Complementing Condition holds for the boundary value problem (53) and (56) with the following weights:

$$
\begin{aligned}
& \left\{t_{j}\right\}_{j=1}^{8}=(1,1,1,1,2,2,2,2), \\
& \left\{s_{i}\right\}_{i=1}^{8}=(0,0,0,0,-1,-1,-1,-1) .
\end{aligned}
$$

- For any smooth right-hand sides satisfying (59) and (60), the problem (57), (58), and (61) has a unique solution $U$; if for $q \geq 0, U$ is a solution that belongs to $\left[H^{q+1}(\Omega)\right]^{3} \times \widetilde{H}^{q+1}(\Omega) \times\left[H^{q+2}(\Omega) \cap H_{0}^{1}(\Omega)\right]^{3}$, then there exists $a$ constant $C>0$ such that

$$
\|\boldsymbol{\omega}\|_{q+1}+\|p\|_{q+1}+\|\mathbf{u}\|_{q+2} \leq C\left(\left\|\mathbf{f}_{1}\right\|_{q}+\left\|f_{2}\right\|_{q}+\left\|\mathbf{f}_{3}\right\|_{q+1}+\left\|f_{4}\right\|_{q+1}\right) .
$$

- The estimate (62) can be extended to negative regularity indices $q$.

5.1. The weighted least squares functional in 3D. For the velocity boundary conditions (58), the mesh-dependent least squares functional $\mathscr{J}^{h}(U)$ in three dimensions is given by

$$
\begin{aligned}
\mathscr{J}^{h}(U)= & \left\|\operatorname{curl} \boldsymbol{\omega}+\operatorname{grad} p-\mathbf{f}_{1}\right\|_{0, \Omega}^{2}+\left\|\operatorname{div} \boldsymbol{\omega}-f_{2}\right\|_{0, \Omega}^{2} \\
& +h^{-2}\left\|\operatorname{curl} \mathbf{u}-\omega-\mathbf{f}_{3}\right\|_{0, \Omega}^{2}+h^{-2}\left\|\operatorname{div} \mathbf{u}-f_{4}\right\|_{0, \Omega}^{2} .
\end{aligned}
$$


We consider minimization of (63) over a finite-dimensional subspace $\mathbf{U}^{h}$ of

$$
\left[H^{1}(\Omega)\right]^{3} \times \widetilde{H}^{1}(\Omega) \times\left[H_{0}^{1}(\Omega)\right]^{3} .
$$

The index $d$ is again subject to the condition (40), and with the weights determined in Proposition 5, we find that $d$ should be at least 1 . Then, for $d \geq 1$, we assume that the finite element spaces approximate optimally with respect to $H^{d+t_{j}}(\Omega)$. Finally, let $U^{h}=\left(\omega^{h}, p^{h}, \mathbf{u}^{h}\right)$ denote the minimizer of (63) out of $\mathbf{U}^{h}$. Then, we have the following result.

Theorem 5. Let $-d \leq q \leq-1$. Let the hypotheses of Proposition 5 hold. Then there exists $C>0$ such that

$$
\left\|\boldsymbol{\omega}-\boldsymbol{\omega}^{h}\right\|_{q+1}+\left\|p-p^{h}\right\|_{q+1}+\left\|\mathbf{u}-\mathbf{u}^{h}\right\|_{q+2} \leq C h^{d-q}\left(\|\boldsymbol{\omega}\|_{d+1}+\|p\|_{d+1}+\|\mathbf{u}\|_{d+2}\right) .
$$

Thus, the results in three dimensions are the same as those for two dimensions, and the discussions of $\S \S 2-4$ for the latter case carry over virtually intact to the former case.

\section{NumericAl RESUltS}

We take for our domain the unit square $\Omega=\{0 \leq x \leq 1,0 \leq y \leq 1\}$ and we consider the generalized Stokes equations (2) where $\mathbf{f}_{1}, f_{2}$, and $f_{3}$ are given functions. We consider the two sets of boundary conditions (4) and (5) where $\mathbf{u}^{0}, P^{0}$, and $U^{0}$ are given functions defined on $\Gamma$. We will define the various data functions by choosing an exact solution $U=(\omega, p, \mathbf{u})$ and then substituting into the equations and the boundary conditions.

In our examples we use $\mathrm{BC} 1 \mathrm{~W}$ to label results obtained with the weighted least squares functional for the velocity boundary condition (4). With BC1 we label results for the same boundary condition but obtained when the weights are removed from the functional. Finally, BC2 labels results for the standard least squares method with the boundary condition (5).

Our numerical results were obtained using, for all unknowns, piecewise quadratic finite element spaces based on a uniform triangulation; for nonuniform grids we found virtually the same convergence rates. Hence, we expect that for the velocity boundary conditions convergence rates will be determined according to (49) if we use the weighted least squares functional. Convergence rates for the pressure-normal velocity boundary condition should be as in (31). For a computational study of the accuracy for the unweighted least squares functional we refer to [5].

Our computational results for the pressure-normal velocity boundary condition (5) involve inhomogeneous boundary conditions. In this case we use boundary interpolants of the data in the corresponding finite element spaces in order to define boundary conditions that could be satisfied by the finite element functions. This method of treating the boundary conditions did not introduce a noticeable change in the convergence behavior of the least squares approximations.

Here we only consider computational results for the exact solution given by

$$
\begin{aligned}
u_{1}=u_{2} & =\sin (\pi x) \sin (\pi y), \\
\omega & =\sin (\pi x) \exp (\pi y), \\
p & =\cos (\pi x) \exp (\pi y) .
\end{aligned}
$$



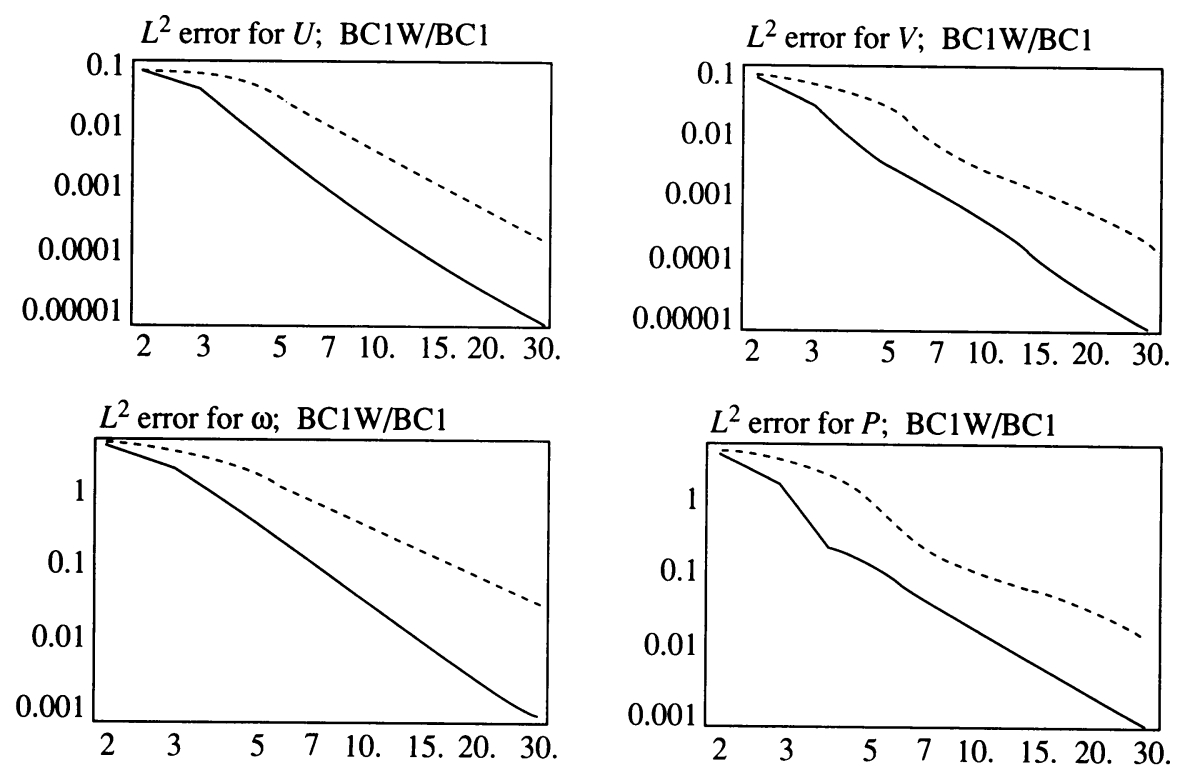

FIGURE 1. $L^{2}$ errors vs. number of grid intervals in each direction. Velocity boundary condition: weighted vs. unweighted functional
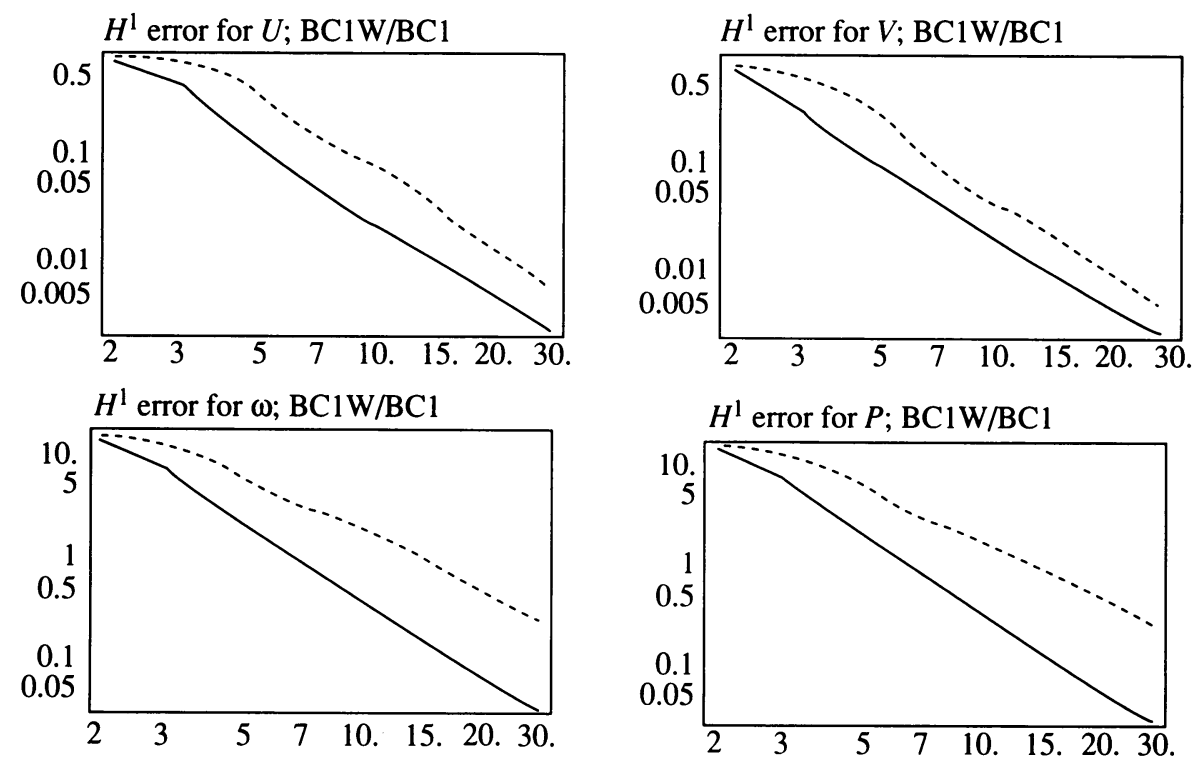

FIGURE 2. $H^{1}$ errors vs. number of grid intervals in each direction. Velocity boundary condition: weighted vs. unweighted functional

The homogeneous velocity boundary condition for this solution can be satisfied exactly, so that the error estimates hold unconditionally. The second reason to choose this solution is that $\omega$ and $p$ are conjugate harmonic functions (see Remark 1 in §2.1.1) with curl $\omega+\operatorname{grad} p=0$, and we expect that the elimination of the weights from the functional will lead to the noticeable reduction in the convergence rates. 

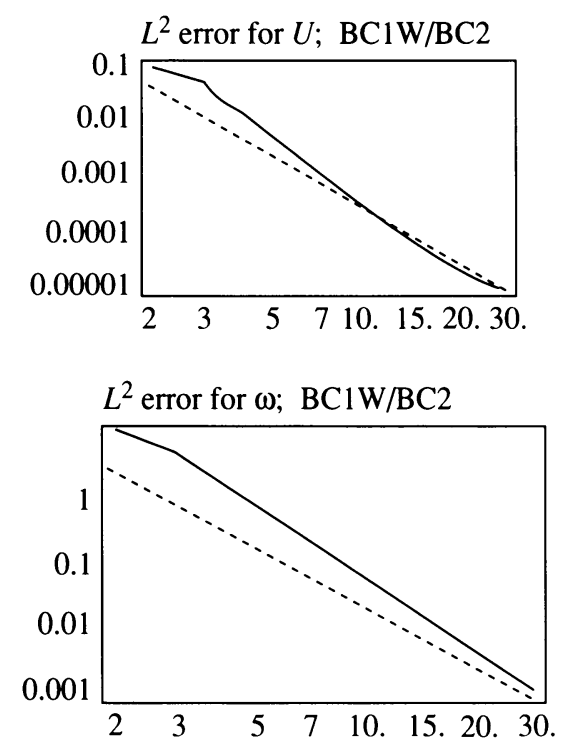
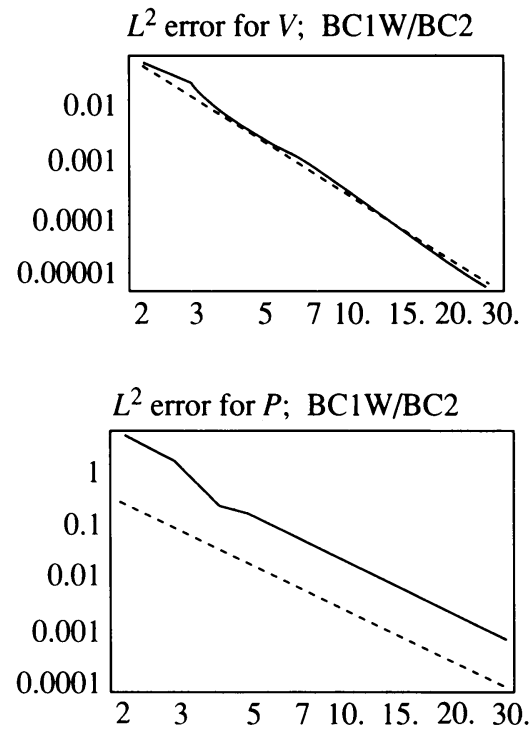

FIGURE 3. $L^{2}$ errors vs. number of grid intervals in each direction. Velocity vs. normal velocity-pressure boundary condition

Figures 1 and 2 give $\log$-log plots of the $L^{2}$ and $H^{1}$ errors, respectively, vs. the number of grid intervals in each direction for a uniform grid spacing. The solid line corresponds to the results obtained with the weighted least squares functional and the dashed line is for the results computed without the weights in the functional. (Note that in the figures, $U=u_{1}$ and $V=u_{2}$.)

In Figures 3 and 4 we compare results obtained with the weighted least squares method for velocity boundary conditions (solid lines) with the results for the standard least squares method for pressure-normal velocity boundary conditions (dashed lines).

The slopes of the curves in Figures 1 to 4 correspond to the rates of convergence; it is evident from the plots presented in Figures 1 and 2 that the addition of the weights to the least squares functional improves the asymptotic convergence rates. From the plots in Figures 3 and 4 one can also infer that asymptotically the convergence rates of the weighted least squares approximations for the velocity boundary condition are identical with the convergence rates for the normal velocity-pressure boundary condition and the standard least squares functional.

In fact, conclusions drawn from Figures 1 to 4 can be supported by computing the slope of least squares straight line fits to the various curves in the figures. The results for these slopes are summarized in Table 1 (next page).

The differences between the rates in the $\mathrm{BC} 1 \mathrm{~W}$ and $\mathrm{BC} 1$ columns suggest that the unweighted least squares results are approximately one order less accurate than the corresponding weighted ones. The nonoptimality of the approximations computed without the weights is best seen in the vorticity component; recall the well-documented fact that methods which use the vorticity as a primary variable often yield very poor approximations; see [18]. 

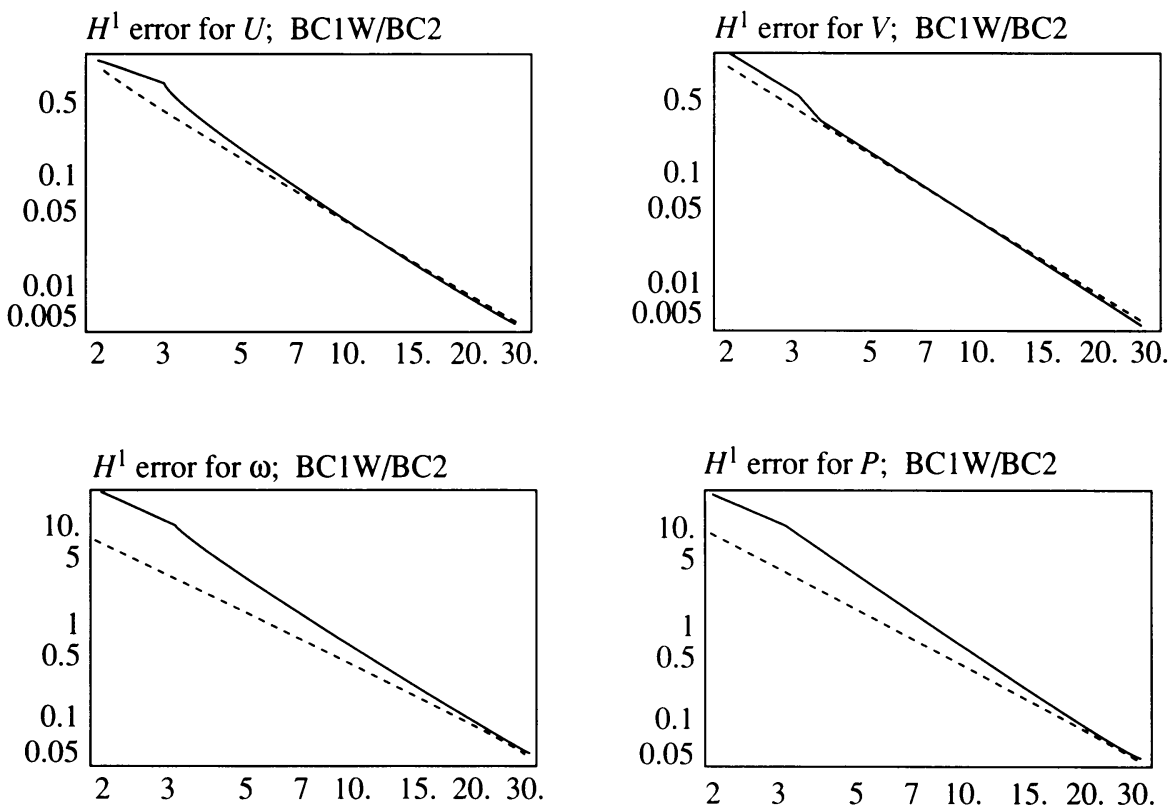

FIGURE 4. $H^{1}$ errors vs. number of grid intervals in each direction. Velocity vs. normal velocity-pressure boundary condition

We used the same degree polynomials based on the same grid for all variables, since that is one of the advantages of the least squares approach. According to the theory of $\S 4$, we could have used one degree lower polynomials, i.e., piecewise linears, for the vorticity and pressure. On the other hand, we draw attention to the fact that the weighted least squares method produces results which exhibit the expected convergence rates for the approximations of the velocity and approximately one order higher rates than is expected from (50) and (51) for the approximations of the vorticity and the pressure. That means all four fields are approximated with the same order although one cannot infer this from the error analysis in $\S 4$. In fact, from Table 1 we can see that the rates under the $\mathrm{BC} 1 \mathrm{~W}$ columns are roughly the same as the rates under the $\mathrm{BC} 2$ columns, and for the latter the error estimates (31) indeed imply that all four fields should be approximated with the same order. Currently, we are unable to give a rigorous justification of this fact, and it is not clear whether such justification can be obtained along the same lines as for the error estimates in $\S \S 2-4$. However, based on the computational evidence, one can argue that the

TABLE 1. Rates of convergence of the $H^{1}$ and $L^{2}$ errors in the least squares finite element solution with and without the weights

\begin{tabular}{||c||c|c|c||c|c|c||}
\hline \multicolumn{1}{|c||}{} & \multicolumn{3}{c||}{$L^{2}$ error rates } & \multicolumn{3}{c||}{$H^{1}$ error rates } \\
\hline Function & BC1W & BC1 & BC2 & BC1W & BC1 & BC2 \\
\hline $\mathbf{u}$ & 3.64 & 2.71 & 3.11 & 2.15 & 2.03 & 2.04 \\
\hline $\mathbf{v}$ & 3.31 & 2.37 & 3.10 & 2.10 & 2.06 & 2.02 \\
\hline$\omega$ & 3.57 & 2.20 & 3.00 & 2.35 & 1.64 & 1.93 \\
\hline$p$ & 3.11 & 2.34 & 2.98 & 2.37 & 1.64 & 1.97 \\
\hline
\end{tabular}


weighted least squares method can possibly take advantage of the better finite element spaces used for the approximations of the vorticity and the pressure.

\section{ACKNOWLEDGMENT}

The authors wish to thank the referee whose detailed remarks and suggestions helped to improve the content and the style of the paper. In particular, the permutation under which $\mathscr{L}$ becomes selfadjoint, and some ideas which led to a shorter proof of Theorem 2 , were pointed out to us by the referee.

\section{BIBLIOGRAPHY}

1. S. Agmon, A. Douglis, and L. Nirenberg, Estimates near the boundary for solutions of elliptic partial differential equations satisfying general boundary conditions. II, Comm. Pure Appl. Math. 17 (1964), 35-92.

2. A. Aziz, R. Kellogg, and A. Stephens, Least-squares methods for elliptic systems, Math. Comp. 44 (1985), 53-70.

3. M. A. Behr, L. P. Franca, and T. E. Tezduyar, Stabilized finite element methods for the velocity-pressure-stress formulation of incompressible flows, Comput. Methods Appl. Mech. Engrg. 104 (1993), 31-48.

4. P. Bochev, Least-squares methods for Navier-Stokes equations, Ph.D. Thesis, Virginia Polytechnic Institute and State University, Blacksburg, VA, 1994.

5. P. Bochev and M. Gunzburger, Accuracy of least-squares methods for the Navier-Stokes equations, Comput. \& Fluids 22 (1993), 549-563.

6. J. H. Bramble and A. H. Schatz, Least-squares methods for 2 mth order elliptic boundary value problems, Math. Comp. 25 (1971), 1-32.

7. F. Brezzi, J. Rappaz, and P.-A. Raviart, Finite-dimensional approximation of nonlinear problems, Part I: Branches of nonsingular solutions, Numer. Math. 36 (1980), 1-25.

8. C.-L. Chang, A mixed finite element method for the Stokes problem: an acceleration-pressure formulation, Appl. Math. Comput. 36 (1990), 135-146.

9. __ Least-squares finite-element method for incompressible flow in 3-D (to appear).

10. C.-L. Chang and M. Gunzburger, A finite element method for first order systems in three dimensions, Appl. Math. Comput. 23 (1987), 171-184.

11. C.-L. Chang and B.-N. Jiang, An error analysis of least-squares finite element methods of velocity-vorticity-pressure formulation for the Stokes problem, Comput. Methods Appl. Mech. Engrg. 84 (1990), 247-255.

12. C.-L. Chang and L. Povinelli, Piecewise linear approach to the Stokes equations in 3-D (to appear).

13. P. Ciarlet, Finite element method for elliptic problems, North-Holland, Amsterdam, 1978.

14. G. Fix, M. Gunzburger, R. Nicolaides, and J. Peterson, Mixed finite element approximations for the biharmonic equations, Proc. 5th Internat. Sympos. on Finite Elements and Flow Problems (J. T. Oden, ed.), University of Texas, Austin, 1984, pp. 281-286.

15. L. P. Franca and R. Stenberg, Error analysis of some Galerkin least-squares methods for the elasticity equations, SIAM J. Numer. Anal. 28 (1991), 1680-1697.

16. V. Girault and P.-A. Raviart, Finite element methods for Navier-Stokes equations, Springer, Berlin, 1986.

17. M. Gunzburger, Finite element methods for viscous incompressible flows, Academic Press, Boston, 1989.

18. M. Gunzburger, M. Mundt, and J. Peterson, Experiences with computational methods for the velocity-vorticity formulation of incompressible viscous flows, Computational Methods in Viscous Aerodynamics (T. K. S. Murthy and C. A. Brebbia, eds.), Elsevier, Amsterdam, 1990, pp. 231-271. 
19. B.-N. Jiang, A least-squares finite element method for incompressible Navier-Stokes problems, Internat. J. Numer. Methods Fluids 14 (1992), 943-859.

20. B.-N. Jiang and C. Chang, Least-squares finite elements for the Stokes problem, Comput. Methods Appl. Mech. Engrg. 78 (1990), 297-311.

21. B.-N. Jiang, T. Lin, and L. Povinelli, Large-scale computation of incompressible viscous flow by least-squares finite element methods, Comput. Methods Appl. Mech. Engrg. (to appear).

22. B.-N. Jiang and L. Povinelli, Least-squares finite element method for fluid dynamics, Comput. Methods Appl. Mech. Engrg. 81 (1990), 13-37.

23. B.-N. Jiang and V. Sonnad, Least-squares solution of incompressible Navier-Stokes equations with the p-version of finite elements, NASA TM 105203 (ICOMP Report 91-14), NASA, Cleveland, 1991.

24. D. Lefebvre, J. Peraire, and K. Morgan, Least-squares finite element solution of compressible and incompressible flows, Internat. J. Numer. Methods Heat Fluid Flow 2 (1992), 99-113.

25. J.-L. Lions and E. Magenes, Nonhomogeneous elliptic boundary value problems and applications, Vol. I, Springer, Berlin, 1972.

26. M. Renardy and R. Rogers, Introduction to partial differential equations, Springer, Berlin, 1993.

27. R. Roitberg and Z. Seftel, A theorem on homeomorphisms for elliptic systems and its applications, Math. USSR Sb. 7 (1969), 439-465.

28. L. Tang and T. Tsang, A least-squares finite element method for time-dependent incompressible flows with thermal convection, Internat. J. Numer. Methods Fluids (to appear).

29. W. Wendland, Elliptic systems in the plane, Pitman, London, 1979.

Department of MATHEMATICS AND INTERdisciplinary Center for APPlied Mathematics, Virginia Tech, Blacksburg, Virginia 24061-0531

E-mail address, P. Bochev: bochev@next.icam.vt.edu

E-mail address, M. Gunzburger: gunzburger@vtccl.cc.vt.edu 